\title{
Organoids from human tooth showing epithelial stemness phenotype and differentiation potential
}

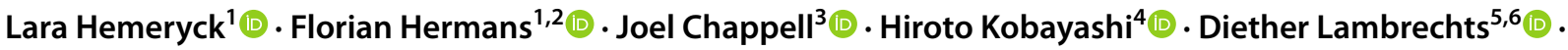 \\ Ivo Lambrichts ${ }^{2}$ (I) . Annelies Bronckaers ${ }^{2}$ (D) Hugo Vankelecom ${ }^{1}[$
}

Received: 7 October 2021 / Revised: 27 January 2022 / Accepted: 1 February 2022 / Published online: 26 February 2022

(c) The Author(s) 2022

\begin{abstract}
Insight into human tooth epithelial stem cells and their biology is sparse. Tissue-derived organoid models typically replicate the tissue's epithelial stem cell compartment. Here, we developed a first-in-time epithelial organoid model starting from human tooth. Dental follicle (DF) tissue, isolated from unerupted wisdom teeth, efficiently generated epithelial organoids that were long-term expandable. The organoids displayed a tooth epithelial stemness phenotype similar to the DF's epithelial cell rests of Malassez (ERM), a compartment containing dental epithelial stem cells. Single-cell transcriptomics reinforced this organoid-ERM congruence, and uncovered novel, mouse-mirroring stem cell features. Exposure of the organoids to epidermal growth factor induced transient proliferation and eventual epithelial-mesenchymal transition, highly mimicking events taking place in the ERM in vivo. Moreover, the ERM stemness organoids were able to unfold an ameloblast differentiation process, further enhanced by transforming growth factor- $\beta$ (TGF $\beta$ ) and abrogated by TGF $\beta$ receptor inhibition, thereby reproducing TGF $\beta$ 's known key position in amelogenesis. Interestingly, by creating a mesenchymal-epithelial composite organoid (assembloid) model, we demonstrated that the presence of dental mesenchymal cells (i.e. pulp stem cells) triggered ameloblast differentiation in the epithelial stem cells, thus replicating the known importance of mesenchyme-epithelium interaction in tooth development and amelogenesis. Also here, differentiation was abrogated by TGF $\beta$ receptor inhibition. Together, we developed novel organoid models empowering the exploration of human tooth epithelial stem cell biology and function as well as their interplay with dental mesenchyme, all at present only poorly defined in humans. Moreover, the new models may pave the way to future tooth-regenerative perspectives.
\end{abstract}

Keywords Tooth $\cdot$ Organoids $\cdot$ Stem cells $\cdot$ Ameloblasts $\cdot$ Assembloids $\cdot$ TGF $\beta$

Hugo Vankelecom

hugo.vankelecom@kuleuven.be

1 Laboratory of Tissue Plasticity in Health and Disease, Cluster of Stem Cell and Developmental Biology,

Department of Development and Regeneration, Leuven Stem Cell Institute, KU Leuven (University of Leuven), Leuven, Belgium

2 Faculty of Medicine and Life Sciences, Biomedical Research Institute (BIOMED), UHasselt (Hasselt University), Diepenbeek, Belgium

3 Department of Bioinformatics, Bit.bio, Babraham Research Campus, Cambridge, UK

4 Department of Anatomy and Structural Science, Faculty of Medicine, Yamagata University, Yamagata, Japan

5 VIB - Center for Cancer Biology, Leuven, Belgium

6 Laboratory for Translational Genetics, Department of Human Genetics, KU Leuven, Leuven, Belgium

\section{Introduction}

Teeth play essential roles in food mastication and speech. Moreover, tooth physiology is more and more highlighted to impact body health and disease [1-3]. In contrast to the wealth of knowledge on tooth development, homeostatic maintenance and repair in rodents, tooth biology remains far from understood in humans [4]. Although stem cells of the mesenchymal compartments such as dental pulp and periodontal ligament (PDL) have substantially been characterized, knowledge on human tooth epithelial stem cells regarding presence, phenotype and biological function is scarce [4]. Some indications for their existence have been found in the epithelial cell rests of Malassez (ERM), a network of epithelial cells that is present in the dental follicle (DF) which encloses unerupted teeth and upon tooth eruption remains present in the PDL around the root [5]. These nests 
(a)
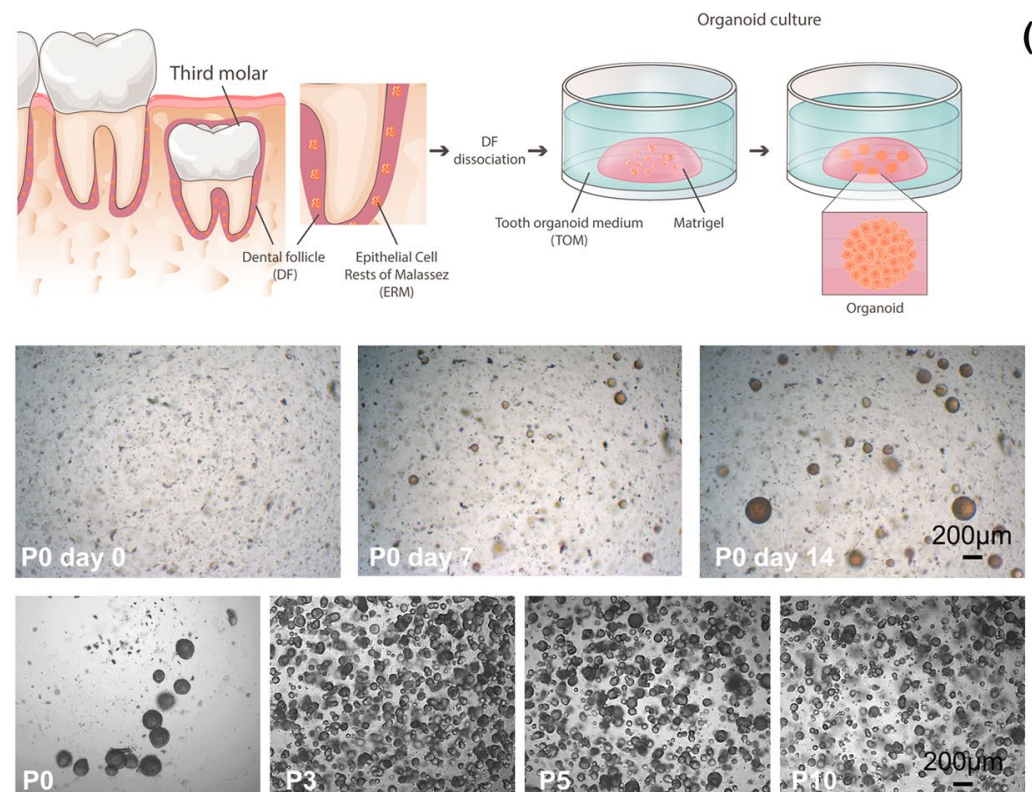

(c)

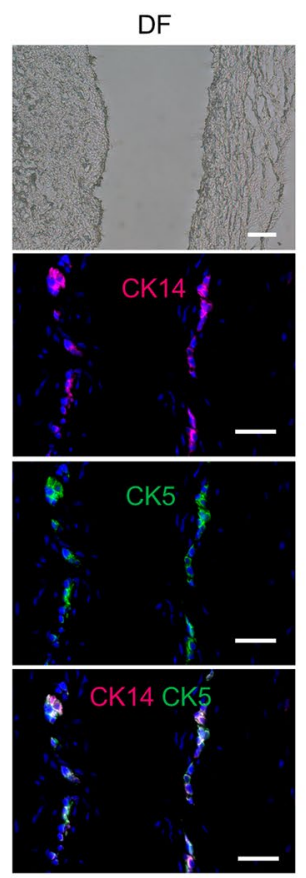

(e)
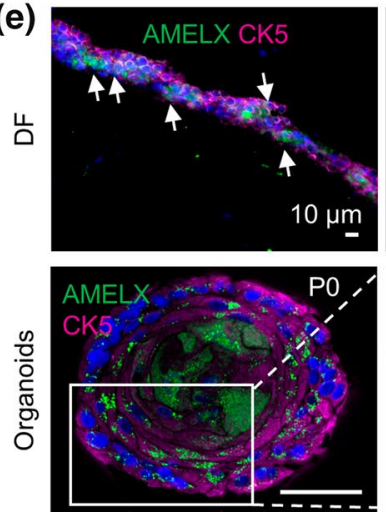

Organoids
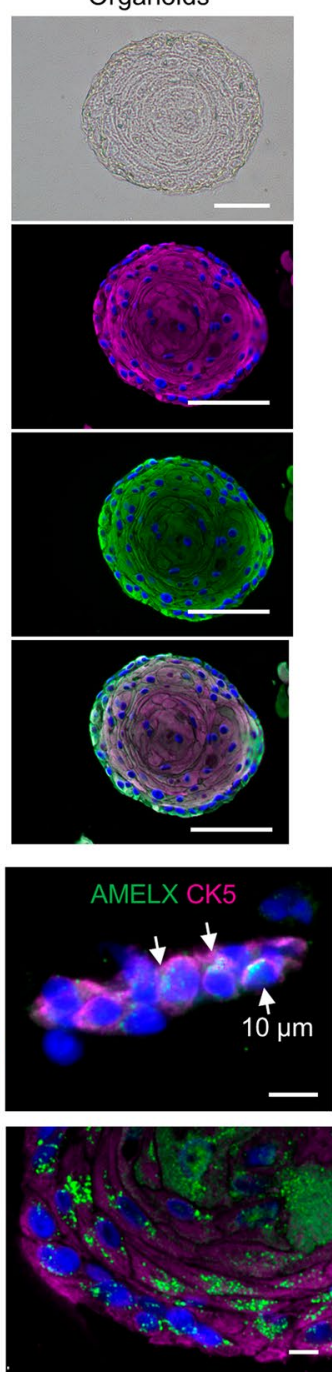

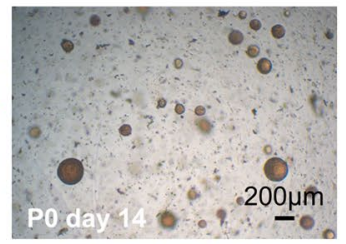

(b)
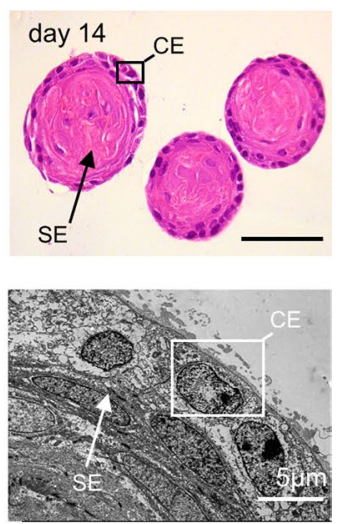

(d)

CK5 CD44
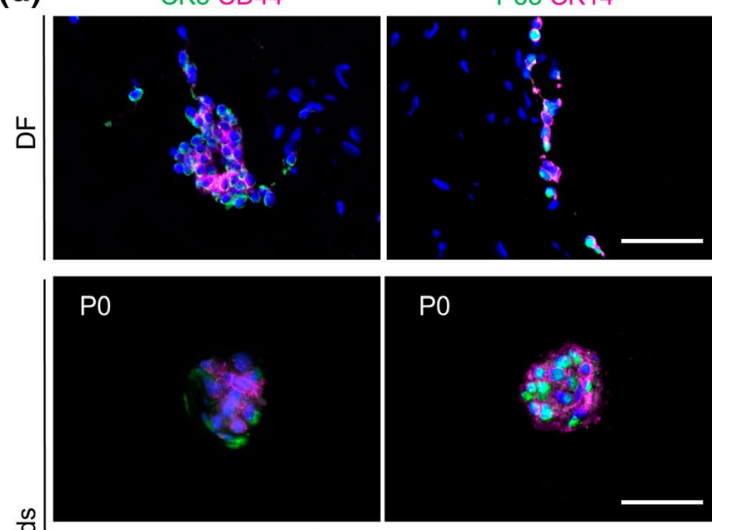

产
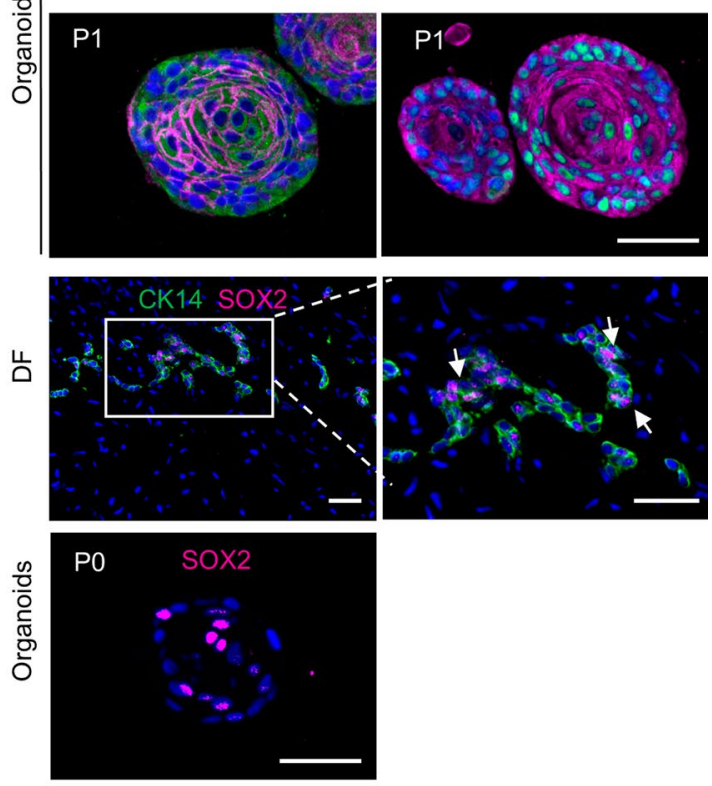
४Fig. 1 Establishment of organoids from human dental follicle. a Schematic of organoid culture set-up. Progressing development of organoid structures after seeding dissociated dental follicle (DF) in tooth organoid medium (TOM) (passage $0, \mathrm{P} 0$ ), and robust passageability (brightfield pictures of indicated P). b Histological (H\&E) and ultrastructural (TEM) analyses of tooth organoids grown in TOM for 14 days. Box and arrow indicate cuboidal epithelium (CE) and squamous epithelium (SE), respectively. c-e Brightfield phase-contrast images and immunofluorescence staining pictures for markers as indicated, of primary DF tissue and full-grown (day-14) organoids. Arrows indicate double-positive cells of indicated markers. Boxed areas are enlarged. DAPI (blue) was used to label nuclei. Scale bars: $50 \mu \mathrm{m}$, unless indicated otherwise

of epithelial cells express some stem cell-associated markers, and may play a role in regeneration of enamel and PDL following injury and inflammation, although repair capacity appears limited in postnatal life [5, 6]. During tooth development, enamel is formed by epithelial cells called ameloblasts [4]. It has been reported that ERM-derived cells, when cocultured with dental pulp stem cells (DPSCs), can differentiate into ameloblast-like cells [7]. However, 2D-cultured ERM cells show highly limited growth capacity and rapid loss of phenotype [8-12].

A powerful method to in vitro grow and expand tissue epithelial stem cells is provided by the organoid technology [13-17]. Organoids are 3D cell constructs that self-develop by proliferative expansion from tissue's epithelial stem cells when the dissociated primary tissue sample (containing the stem cells as single cells or contained within cell clusters) is seeded into an extracellular matrix (ECM)-mimicking scaffold (typically, Matrigel) and cultured in a defined cocktail of growth factors replicating stem cell niche signaling (if known) and/or tissue embryogenesis. Among others, activation of wingless-type MMTV integration site (WNT) and epidermal growth factor (EGF) signaling are typically needed [14-16]. Resultant organoids duplicate the epithelial stem cell compartment of the tissue of origin in molecular phenotype and functional characteristics, and can generate differentiated tissue cell types under specified culture conditions [13-15]. As an important asset, organoid cultures can be serially expanded (passaged) without loss of characteristics, thereby providing a robust and faithful source of the primary tissue's epithelial stem cells and overcoming their generally limited availability and culture-ability. Typically, epithelial organoid models are established without the need for prior isolation of the epithelial (stem) cells from the dissociated whole-tissue sample since the accompanying mesenchymal cells do not thrive in the specific culture conditions used and are swiftly lost at culture and passaging $[13,14,16,17]$. Although meanwhile derived from numerous organs, epithelial organoids have not been established yet from human tooth $[18,19]$. A previous study reported that ERM cells, seeded in Matrigel, grew as 'organoids' [8]. However, these structures were not deeply characterized and did not adhere to the current hallmarks of tissue-derived organoids [13-17] such as (clonal) derivation and expansion from epithelial tissue stem cells under WNT-promoting conditions, and robust and long-term expandability. Other studies described the construction of bioengineered 3D dental structures, however, only from animal origin (mouse, rat, $\mathrm{dog}, \mathrm{pig}$ ) at embryonic or neonatal age and non-expandable $[18,20-22]$.

Here, we report the successful establishment of long-term expandable organoid cultures starting from human tooth (i.e. from the DF of third molars). The organoids show epithelial stemness characteristics mirroring ERM stem cells, and display ameloblast differentiation property reinforced by the presence of TGF $\beta$ or dental mesenchymal cells, thereby recapitulating ERM/dental epithelial stem cell (DESC) features and known in vivo processes. The new organoid models provide a valuable research tool to explore human tooth epithelial stem cell biology and epithelium-mesenchyme interplay, at present only poorly understood, thereby paving the way to unraveling their roles in tooth homeostasis and potential repair. Moreover, the tractable biological tooth stem cell structures represent an appealing step toward dental regenerative replacement prospects.

\section{Results}

\section{Organoids can be established from human dental follicle}

To develop epithelial organoids starting from human tooth, the DF, known not only to encompass a large mesenchymal component but also the small epithelial ERM compartment [5], was isolated from unerupted third molars (wisdom teeth) extracted from adolescent patients (Fig. 1a). After tissue dissociation, the epithelial-mesenchymal cell mixture, comprising single cells and cell clusters, was embedded in Matrigel and cultured in a precisely defined medium. Organoids are typically established using a cocktail of growth and regulatory factors active in the tissue's epithelial stem cell niche. In case niche signals are unresolved, factors with a key role in the tissue's embryonic development are applied [13-17]. Hence, since the human tooth epithelial stem cell niche is undefined, we tested growth and signaling factors shown to play a role in tooth development, including sonic hedgehog ( $\mathrm{SHH})$, fibroblast growth factors (FGFs) and insulin-like growth factor-1 (IGF1) [23, 24]. We started with a medium containing these factors as well as generic organoid growth factors (such as WNT activators, nicotinamide, BMP inhibitor and p38 MAPK inhibitor) [13-16, 25 ] and assessed the essentiality of individual factors by omitting. The BMP inhibitor (Noggin), p38 MAPK inhibitor (SB202190), WNT activator (R-spondin 1 (RSPO1)), IGF1, 
(a)

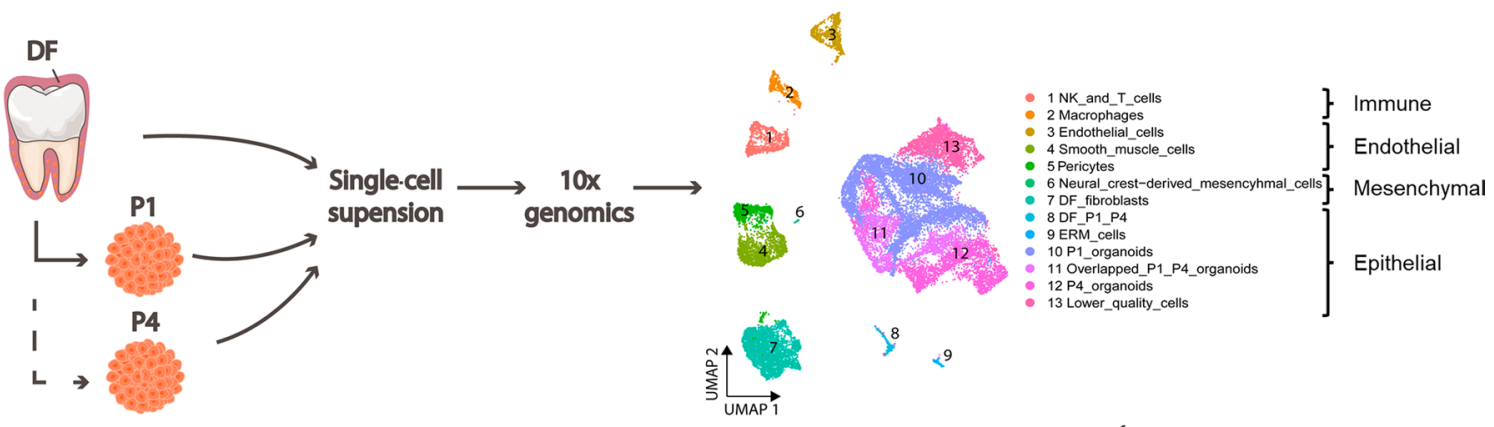

(b)

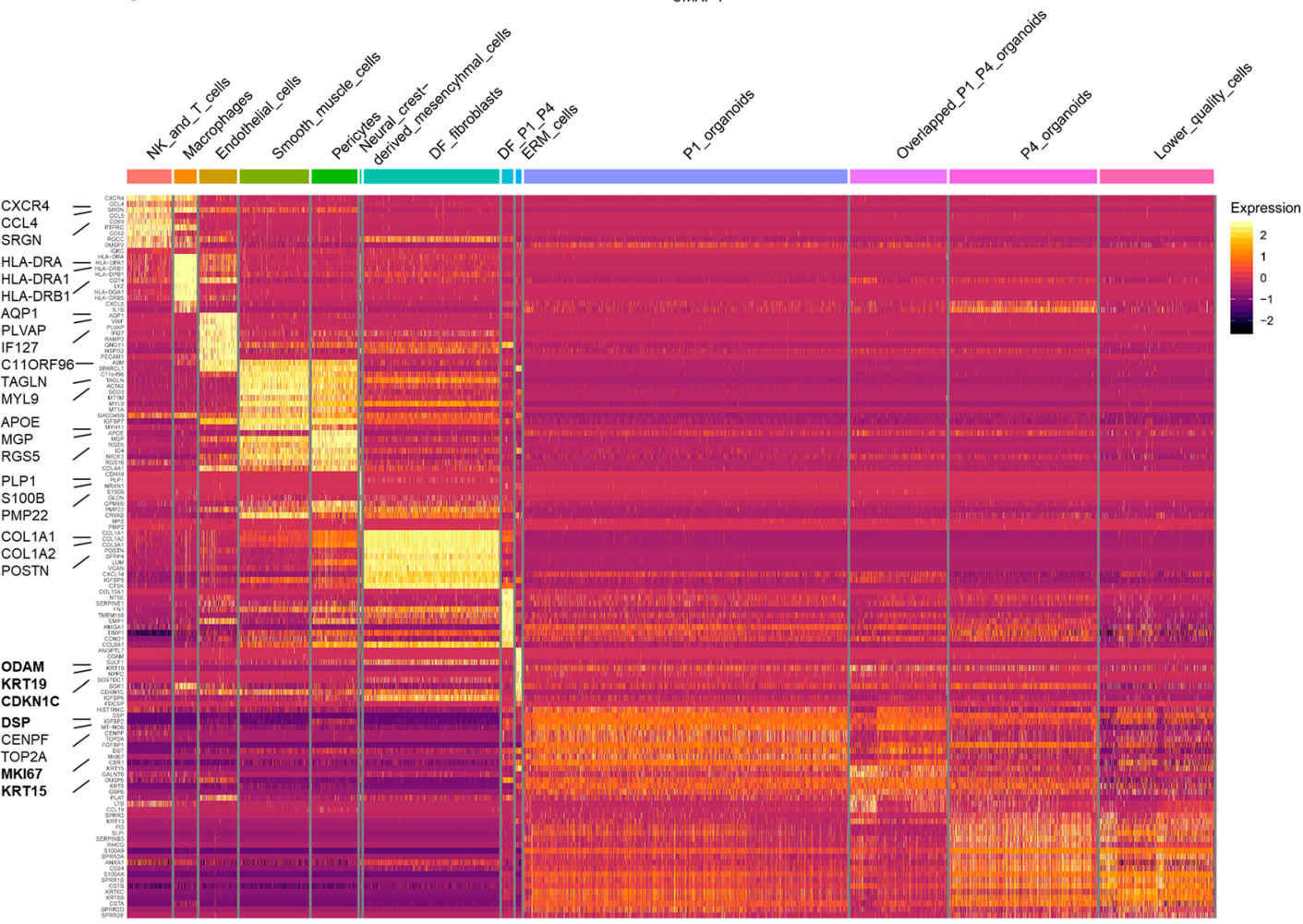

(c)

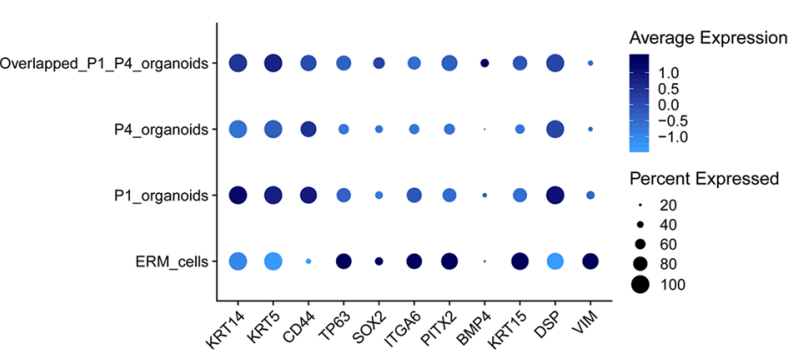

(d) TP63

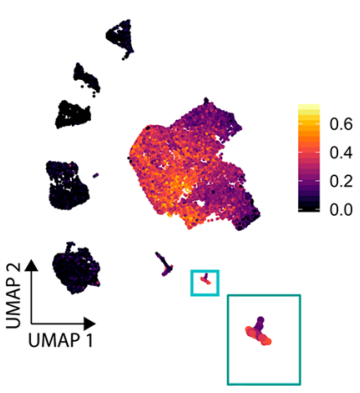

PITX2
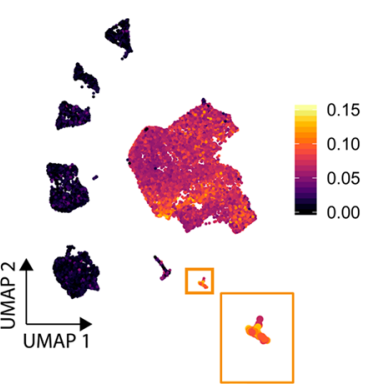

EGR1

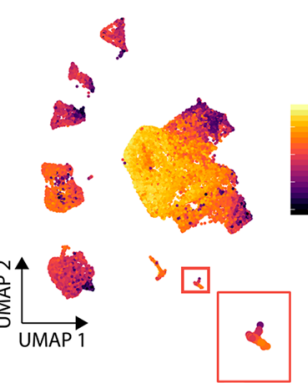

ATF3
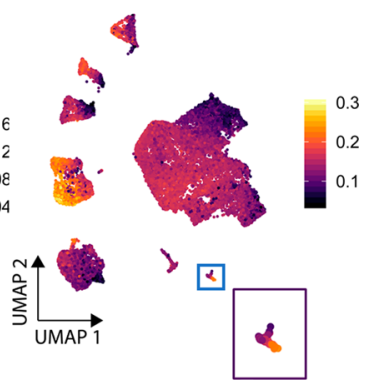
4 Fig. 2 Single-cell transcriptomic profiling of primary DF and corresponding organoids. a Experimental overview of the scRNA-seq analysis. UMAP plot of the annotated cell clusters in the integrated DF-organoid dataset. DF, dental follicle; ERM, Epithelial Cell Rests of Malassez; NK, natural killer cells. b Heatmap displaying the scaled expression of the top 10 differentially expressed genes (DEGs) per cluster. Genes specifically described in the text are highlighted in bold. c Dot plot displaying the percentage of cells (dot size) expressing indicated marker genes with average expression levels (color intensity) (see scales) in the ERM and organoid clusters. d Indicated regulons projected on UMAP plot. The ERM cluster is magnified at the bottom. e Immunofluorescence staining for markers as indicated in primary DF tissue and organoids at specified time points. DAPI (blue) was used to label nuclei. f Significant (FDR $\leq 0.05)$ DEGbased GO terms enriched in ERM versus $\mathrm{P} 1$ organoids (top) or in P1 and $\mathrm{P} 4$ organoids together versus ERM (bottom). g Violin plots showing gene expression level of indicated stemness markers in P1 and P4 organoids. Immunofluorescence staining of P1 and P4 organoids for the indicated markers. DAPI (blue) was used to label nuclei. Scale bars: $50 \mu \mathrm{m}$

SHH, nicotinamide, and FGFs (FGF2, FGF8, FGF10) were all evidently needed for efficient organoid formation (Supplementary Fig. 1a). Eventually, this systematic evaluation led to defining an optimized medium (further referred to as tooth organoid medium or TOM; Supplementary Table 1), allowing to develop organoid lines from DF samples at $100 \%$ efficiency (Supplementary Table 2).

Organoid structures gradually developed in 2 weeks' time (Fig. 1a; passage 0 (P0)), growing out of cell clusters (typically $4-8$ cells wide by 20 cells long, similar to the ERM cell groups present in the primary DF tissue; Supplementary Fig. 1b,c) [5, 26], or of single cells, indicating the capability of clonal development (Supplementary Fig. 1b). Importantly, the organoids were amenable to long-term expansion, at present for more than 10 consecutive passages (i.e. 5 months) (Fig. 1a). At passaging, the organoids were dissociated into single cells and organoid structures efficiently re-grew. When starting from a mixture of cells dissociated from either $\mathrm{eGFP}^{+}$or $\mathrm{eGFP}^{-}$organoid cultures, the organoids that reformed were homogeneously fluorescent or non-fluorescent, suggesting clonal regrowth at passaging (Supplementary Fig. 1d). Mesenchymal cells, also present in the dissociated DF cell mixture, adhered to the bottom of the culture plate following sample seeding (P0; Supplementary Fig. 1e), and were swiftly lost at passaging in the standard, epithelial-favoring organoid culture conditions used (P1; Supplementary Fig. 1e). During a single-passage 14-day culture period, organoids progressively increased in size while the proportion of proliferating $\left(\mathrm{KI}_{6}{ }^{+}\right)$cells gradually decreased and the fraction of apoptotic (cleavedcaspase $3, \mathrm{CC}^{+}$) cells slightly enhanced, although to only low levels which remained invariable over different passages (as determined in full-grown day-14 organoids) (Supplementary Fig. 1f,g). Full-grown organoid size also remained constant over passaging, after a first significant increase from P0 to P1 (Supplementary Fig. 1g). Within individual passages, the organoids displayed considerable size homogeneity (Supplementary Fig. 1g). Finally, organoid cultures could be reconstituted after cryopreservation, and were also establishable from the DF of already erupted wisdom teeth (Supplementary Fig. 1h).

The developed organoid structures displayed a dense morphology (Fig. 1a,b), showing an outer border of stratified cuboidal epithelium (CE) with cells displaying a high nucleo-cytoplasmic ratio, and an adjoining stratified squamous epithelium (SE; Fig. 1b). In DF tissue, epithelial cells with high nucleo-cytoplasmic ratio are present in the ERM (Supplementary Fig. 1c) [5]. In further analogy, the ERM markers cytokeratin (CK) 14 and CK5 [27] were detected in the organoids, as they are observed in compartments of the original DF tissue (Fig. 1c). Importantly, the mesenchymal (fibroblast) marker CD90 (Thy-1 cell surface antigen, THY1), which is observed in compartments of the original DF tissue was not detected in the organoids (Supplementary Fig. 1i), indicating the absence of pure mesenchymal cells in the (epithelial) organoids. Previous studies proposed that the ERM contains DESCs, among others marked by CD44 and P63 [12, 27, 28]. Interestingly, these markers, indeed observed in the primary DF tissue (Fig. 1d), were also detected in the derived organoids, both at initial formation (P0) and after passaging (P1; Fig. 1d). Moreover, the organoids and the native DF tissue expressed SOX2 (Fig. 1d), a well-known marker of DESCs in mouse [29-32] and detected in epithelium of developing teeth in humans [4]. Finally, the organoids showed prominent gene expression of the proposed ERM stem cell marker integrin- $\alpha 6(I T G \alpha \sigma)[8$, 28], as well as of factors playing an important role in embryonic development of the dental epithelium (i.e. paired like homeodomain 2 (PITX2) [33] and bone-morphogenetic protein $4(B M P 4)$ ) [34], all markers also detected in the original DF tissue (Supplementary Fig. 1j). Finally, we also observed expression of amelogenin (AMELX) in both primary tissue (more in particular in the $\mathrm{CK} 5^{+} \mathrm{ERM}$ region of the DF) and derived organoids (Fig. 1e). AMELX is not only a main constituent of enamel matrix but also a suggested marker of ERM cells (described in rat) [6, 35-37]. Proposed functions of this ERM-produced AMELX include maintenance of PDL space [38] and stimulation of cementoblast differentiation $[39,40]$. Of note, AMELX localization can show a punctuated pattern, being present in vesicles and/or secreted in the extracellular space [41], which is also observed here (Fig. 1e).

Taken together, epithelial organoids can be established from human tooth-derived DF, displaying an ERM-mirroring, stemness expression phenotype and possessing robust long-term expandability. 
(e)

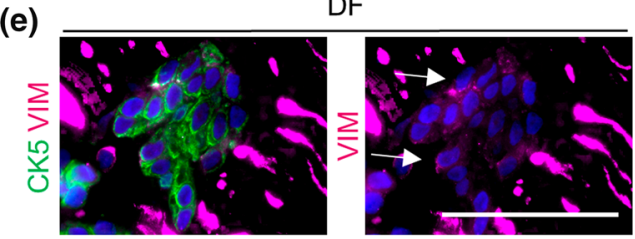

Organoids (P0)

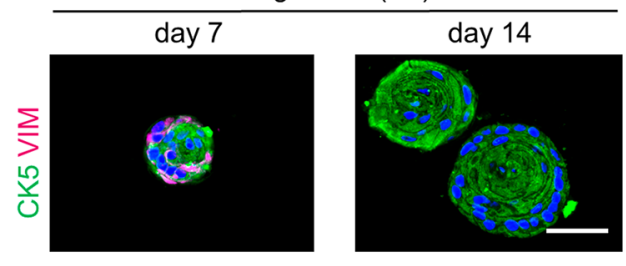

Organoids (P4)

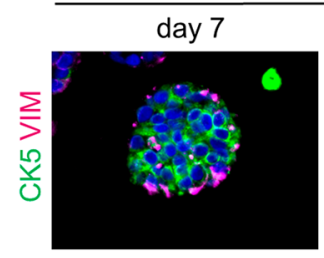

(g)

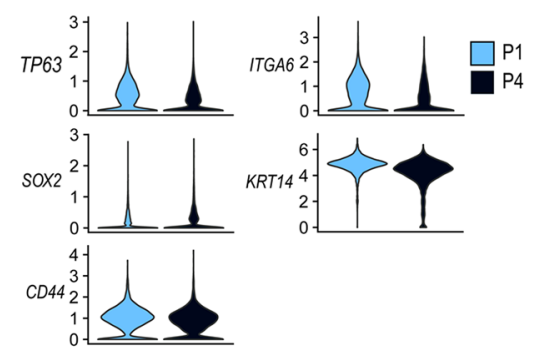

(f)

GO Analysis

ERM vs P1
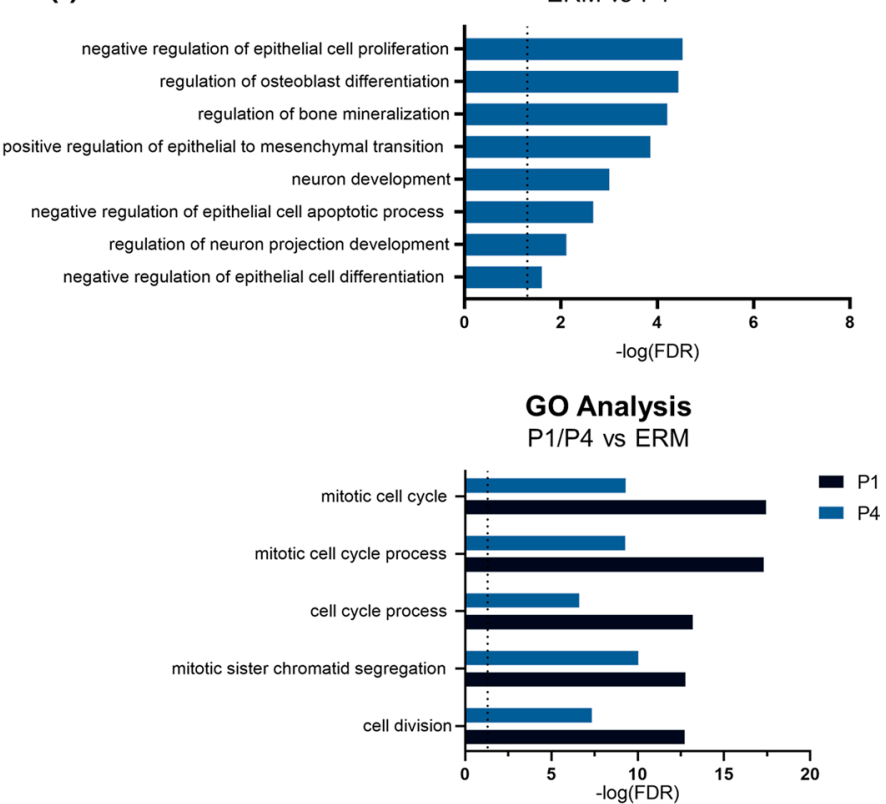

P63 CK14

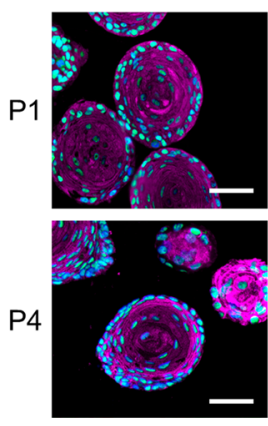

SOX2

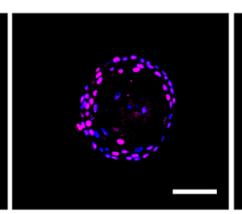

CD44

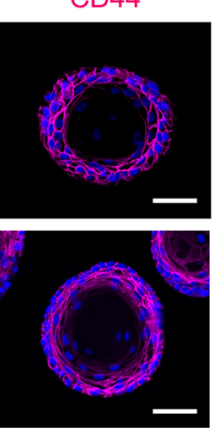

Fig. 2 (continued)

\section{Single-cell transcriptomics reinforces the organoid-ERM congruence}

To decode the organoids in more granular detail, we applied single-cell RNA-sequencing (scRNA-seq) analysis on DFderived organoids (at P1 and P4) together with their primary tissue (Fig. 2a; Supplementary Table 2). Unsupervised clustering of the aggregate data and visualization using uniform manifold approximation and projection (UMAP) [42] exposed main DF cell-type clusters annotated using reported markers [43] comprising immune, endothelial, mesenchymal and epithelial compartments, and grouped organoid clusters with noticeable overlap of the P1 and P4 cultures (Fig. 2a; Supplementary Fig. 2a; Supplementary Dataset 1). Of note, a cluster of lower quality cells (i.e. with low gene counts) was also discerned (Fig. 2a), not filtered out using the applied quality thresholds (see Methods; Supplementary Fig. 2b). This cluster likely comprises dying cells from the organoids' core, as supported by gene ontology (GO) analysis [44] revealing an enriched 'cell death' biological term (Supplementary Fig. 2c), which conforms to ultrastructural features of apoptotic nuclei and absence of cell organelles in the organoids' center (Supplementary Fig. 2c).

Compiling the clusters' top 10 differentially expressed genes (DEGs; Supplementary Dataset 1) in a heatmap exposed specific expression patterns of the different clusters (Fig. 2b). Several of the reported stem cell and ERM markers (see above and [12, 27, 28, 33-35]) were found expressed in the organoids (Fig. 2c; Supplementary Fig. 2a,d), thus corroborating our observations above on organoid-ERM correspondence. Of note, mesenchymal markers such as fibroblast activation protein alpha $(F A P)$ and collagen type I alpha 1 chain (COL1A1), being present in the DF mesenchymal (fibroblast) cluster, were not detected in the organoid clusters, thereby again demonstrating the absence of pure mesenchymal cells in the (epithelial) organoids (Supplementary Fig. 2d). In further analogy with the transcriptomic organoid-ERM congruence, gene-regulatory network (regulon) 
analysis using pySCENIC [45] (i.e. defining core transcription factors with their positively regulated target genes in single cells) showed high TP63 and PITX2 regulon activity in both organoids and ERM (Fig. 2d). Predicted target genes of the PITX2 regulon include SOX2, TP63, PITX2, KRT5, $K R T 14$, and BMP4 (Fig. 2c). Interestingly, the newly proposed mouse incisor epithelial (stem) cell markers KRT15 and dentin sialoprotein (DSP) [46] were among the top 10 DEGs in the organoid as well as ERM clusters (Fig. 2b,c; Supplementary Fig. 2e). Moreover, high regulon activity of early growth response 1 (EGR1) (marking a transient progenitor population in mouse incisor [43]) was observed in organoid clusters and ERM, while substantial regulon activity of activating transcription factor 3 (ATF3) (recently reported as new mouse incisor epithelial cell marker [46]) also showed up in the organoid and ERM clusters (Fig. 2d). Together, our single-cell transcriptomic analysis uncovered new, mouse-mirroring molecular features for human ERM, at present ill-defined. Intriguingly, we also detected gene expression of the mesenchymal marker vimentin (VIM) in ERM cells (Fig. 2c; Supplementary Fig. 2a,e). In agreement, and in analogy with a previous report [47], we observed co-expression of VIM in ERM $\left(\mathrm{CK}^{+}\right)$cells of the primary DF tissue (Fig. 2e), which was recapitulated in the initiating organoids (Fig. 2e; P0, day 7). These findings may point to a hybrid epithelial/mesenchymal (E/M) nature which has recently been correlated with stemness and active stem cells in several other (developing) tissues (such as human fetal pituitary, intestine, liver, lung) [48, 49]. VIM expression faded during further organoid culturing (Fig. 2e; day 14). However, VIM expression transiently reiterated at each passaging (as shown for P4; Fig. 2e), suggesting that the hybrid E/M (active stem cell) phenotype is re-activated at reseeding. Taken together, our profound single-cell transcriptomic analysis further reinforces the congruence between the DF-derived organoids and the ERM (stem) cells residing in this tooth tissue. To finally corroborate this relationship, we isolated ERM stem cells from DF tissue by FACS based on ITG $\alpha 6$ expression ([8, 28] and Supplementary Fig. 2f), and seeded the cells in organoid-developing conditions. The ITG $\alpha 6^{+}$ERM cells formed organoids whereas ITG $\alpha 6^{-}$cells did not (Supplementary Fig. 2f). Interestingly, the latter culture only showed mesenchymal cells adhering to the bottom of the culture plate which were not observed in the ITG $\alpha 6^{+}$ cell culture (Supplementary Fig. 2f), further supporting that the DF's mesenchymal cells do not contribute to, or make part of, organoid growth.

We further compared the organoids with the ERM by applying GO analysis. Negative regulation of 'epithelial cell proliferation', of 'epithelial cell apoptotic process' and of 'epithelial cell differentiation' are significantly enriched in the ERM versus the organoids (P1) (Fig. 2f; Supplementary Dataset 2, 3, 4a,b) which is in line with the quiescence stemness character of ERM under homeostatic conditions as reported before $[5,7,12]$. For instance, the cell cycle inhibitor CDKN1C is among the top DEGs in the ERM cluster (Fig. 2b). On the other hand, GO terms associated with cell cycle division are enriched in the organoids when compared to ERM (Fig. 2f; Supplementary Dataset 2, 3, $4 \mathrm{c}, \mathrm{d})$. In analogy, gene expression of the proliferation marker MKI67 is prominent in the organoid clusters and absent in the ERM cluster, further corroborated by immunostaining (Fig. 2b; Supplementary Fig. 2g). Also, other proliferation markers such as topoisomerase II alpha (TOP2A) and centromere protein $\mathrm{F}(C E N P F)$ were found almost exclusively expressed in the organoid clusters (Supplementary Fig. $2 \mathrm{~g}$ ). Interestingly, $C E N P F$ was recently discovered in the dental epithelium of the continuously growing mouse incisor [46]. Together, these data provide supportive evidence that the ERM stem cells, being quiescent in vivo, are proliferatively (re-)activated in organoid culture.

In further GO analysis, we found that the biological terms 'regulation of osteoblast differentiation', 'regulation of bone mineralization' and 'regulation of neuron projection development/neuron development' are enriched in the ERM (Fig. 2f; Supplementary Dataset 2, 3, 4a,b), all representing previously proposed biological functions of this specific DF cell compartment $[5,26,50]$. Finally, the higher mesenchymal character of the ERM as compared to full-grown organoids (Fig. 2c,e) is reflected in the enriched 'positive regulation of epithelial-mesenchymal transition' (EMT) term (Fig. 2f), in agreement with higher regulon activity of the EMT-driving transcription factors TWIST1 and ZEB1 in the ERM (Supplementary Fig. 2h; Supplementary Dataset 2, 3, 4a,b).

In general, gene expression signatures of $\mathrm{P} 1$ and $\mathrm{P} 4$ organoids display substantial similarity (Fig. 2b; Supplementary Dataset 5). In particular, expression of stemness markers remains comparable after the additional passaging (Fig. 2g), indicating that the tooth organoids retain their stemness phenotype during expansive culture.

Taken together, our detailed scRNA-seq interrogation demonstrates and reinforces the organoid-ERM stemness relationship and uncovered new molecular fingerprints of human ERM, at present only poorly defined.

\section{EGF induces a proliferative and EMT phenotype in tooth organoids, reminiscent of in vivo events in the ERM}

To establish organoids from primary tissues, supplementation of EGF is generally found quintessential [13-16]. Hence, it is remarkable that DF-derived organoids develop and expand in the absence of exogeneous EGF (TOM; Supplementary Table 1). scRNA-seq mining exposed that the EGF receptor (EGFR) ligands amphiregulin $(A R E G)$ and 
heparin-binding EGF ( $H B-E G F)$, together with $E G F R$, are highly expressed in the organoids (Supplementary Fig. 3a) which may substitute for EGF. In line, blocking the EGFR with cetuximab (added at passaging) compromised organoid growth, resulting in smaller organoids (Supplementary Fig. 3b), thereby revealing the need for endogenous EGFR signaling.

(a) $\mathrm{PO}$
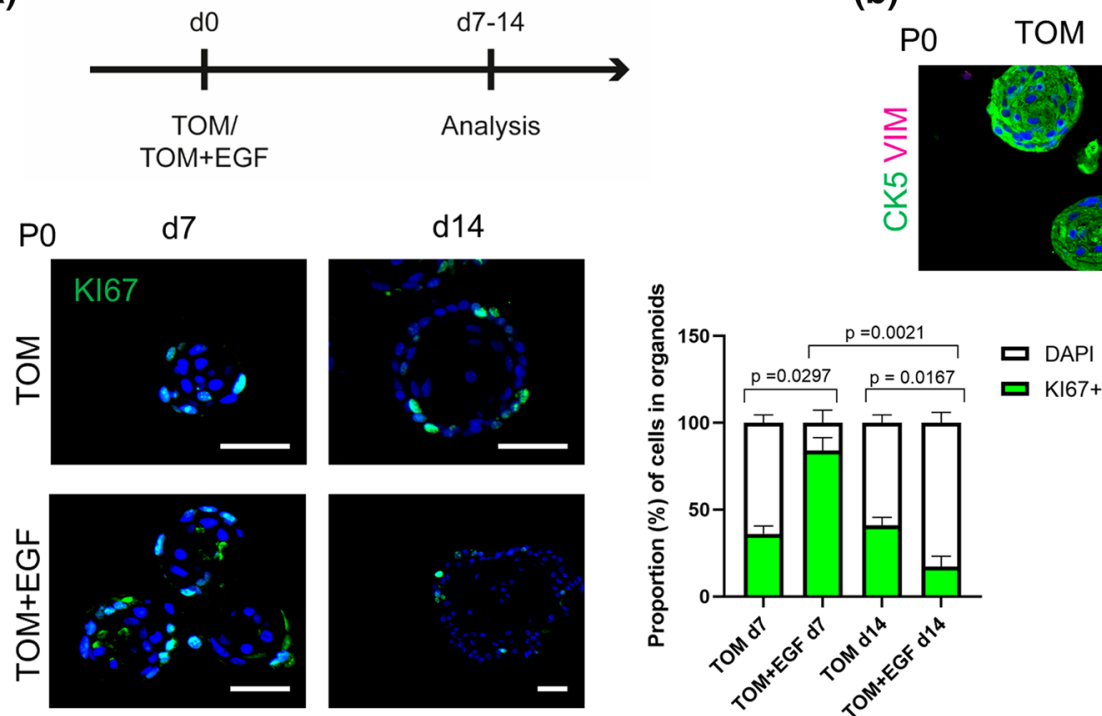

P5

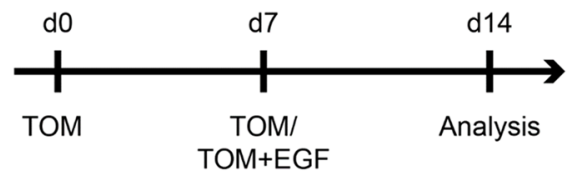

(c)
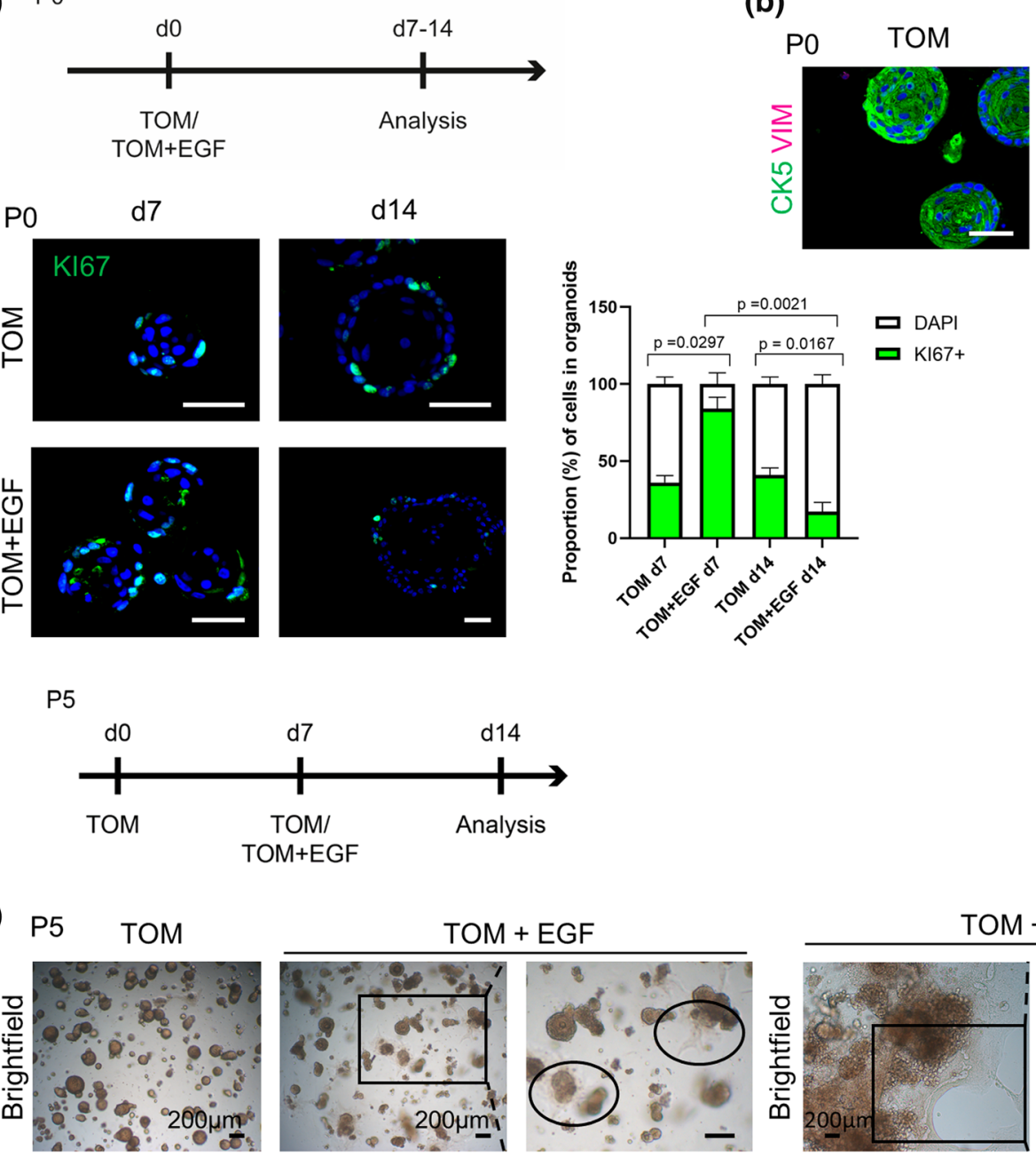

TOM+EGF
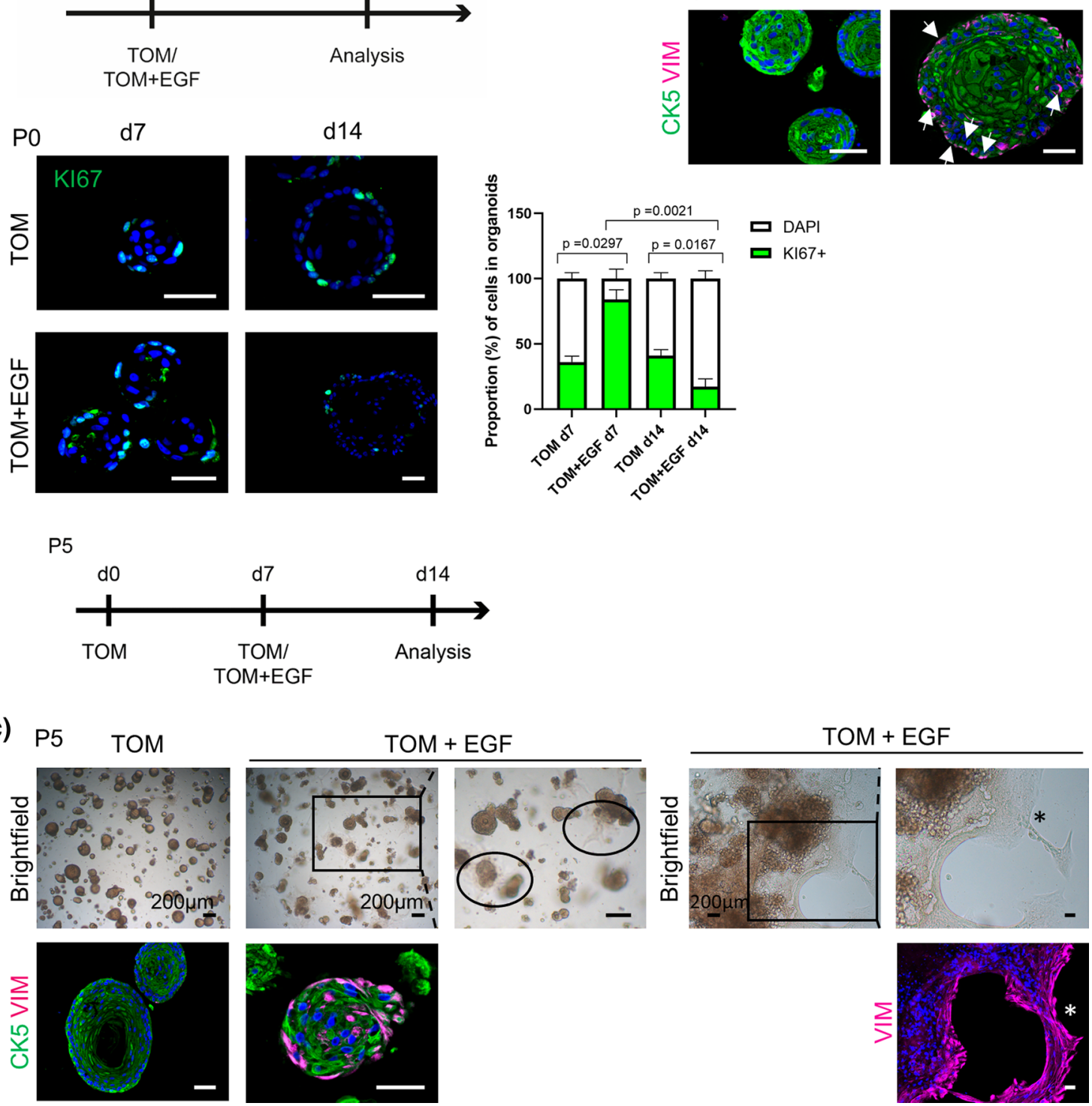

Fig. 3 Effect of EGF on tooth organoid culture. a Timeline of experimental set-up (d, day). Immunofluorescence analysis and quantification of $\mathrm{KI}_{67^{+}}$cells (mean $\pm \mathrm{SEM} ; n=3$ biological replicates) in organoids cultured as indicated. DAPI (blue) was used to label nuclei. b Immunofluorescence staining for the indicated markers of full-grown organoids (day 14; P0) cultured in medium as denoted. Arrows indicate double $\mathrm{VIM}^{+} \mathrm{CK}^{+}$cells. DAPI (blue) was used to label nuclei. c Timeline of experimental set-up. Left part: brightfield pictures of organoid cultures (day 14) as indicated. Boxed area is enlarged. Encircled areas show cell growth at the bottom of the culture plate. Immunofluorescence staining of full-grown organoids (day 14; P5) cultured as indicated for the indicated markers. Right part: brightfield pictures and immunofluorescence (VIM) staining of cells grown at the bottom of the plate (day 14; P5). Boxed area is enlarged. DAPI (blue) was used to label nuclei. Asterisk mark for orientation. Scale bars: $50 \mu \mathrm{m}$, unless indicated otherwise 
Fig. 3a), coinciding with the induction of an EMT process, as supported by the emergence of VIM expression in the organoids' border, found colocalized with epithelial CK5 or P63 in several cells (Fig. 3b; Supplementary Fig. 3c). Moreover, exposing the organoids to EGF after preceding growth and expansion in TOM led to a same process with appearance of $\mathrm{VIM}^{+}$cells (Fig. 3c), as well as migration of cells out of the organoid structures to grow at the bottom of the culture plate, displaying mesenchymal (spindlelike) morphology and VIM expression (Fig. 3c). This motile behavior further underscores the occurrence of EMT, also supported by the upregulated expression of specific mesenchymal/EMT-linked factors (Supplementary Fig. 3d). It has been proposed that EMT induction in the ERM (as, for instance, occurring upon damaging tooth impact) enables the cells to migrate and eventually contribute to regeneration of neighboring tissues [5, 26, 47].

Taken together, adding EGF to the organoids recapitulates functional in vivo behavior of the ERM, thus advancing our new tooth organoid model as an interesting tool to study ERM phenotype and conduct, to date not well understood.

\section{The tooth organoids are amenable to an ameloblast differentiation process}

During tooth development, DESCs give rise to ameloblasts which produce enamel matrix proteins (EMPs) for amelogenesis $[4,52,53]$. It has also been shown that ERM can differentiate into ameloblast(-like) cells $[6,7]$ and produce EMPs [35, 54]. Ameloblast differentiation encompasses a secretory stage with production of the EMPs AMELX and ameloblastin (AMBN), and a maturation stage during which amelotin (AMTN) and odontogenic-ameloblast associated protein (ODAM) are produced [4, 35, 52, 53]. The EMPs are proteolytically cleaved by matrix metalloproteinase 20 (MMP20) and kallikrein (KLK4), typically expressed during the secretory and maturation phase, respectively. Here, we examined whether the DF-derived organoids, possessing an epithelial ERM-stemness phenotype, can be driven into differentiation toward ameloblasts.

Organoids expanded in TOM were switched to a medium previously reported to trigger ameloblast-like differentiation in 2D DESC cultures [41, 55, 56] (referred to as mineralization-inducing medium, MIM; Supplementary Table 3), and analyzed at multiple time points (Fig. 4a). Interestingly, ODAM expression swiftly emerged (from day 2) in the organoids switched to MIM, and increased in intensity while remaining absent in TOM-cultured organoids (Fig. 4a). AMELX protein, being already detected in standard TOM conditions (Fig. 1e), became visually more abundant in MIM-switched organoids from day 8, while remaining more constant in TOM-cultured organoids (Supplementary Fig. 4a). Time-lapse gene expression analysis showed that secretory-stage markers of amelogenesis $[4,35$, 52, 53] mainly increased at day 5 in MIM, while maturation-stage markers $[52,53]$ peaked at day 8-14 (Fig. 4b). In contrast, expression did not change in TOM-cultured organoids (Supplementary Fig. 4b). In parallel with the differentiation process, the stemness phenotype of the organoids dropped showing a fast decline in $\mathrm{SOX}_{2}{ }^{+}$and $\mathrm{TP}^{+}{ }^{+}$cells (Fig. 4b). Interestingly, CK19 expression emerged in MIMswitched organoids (Fig. 4c), in accordance with the known gradual replacement of CK14 by CK19 in differentiating ameloblasts [57]. Moreover, the MIM-cultured organoids displayed calcium deposits (Fig. 4d; Alizarin red S (ARS) staining), supported by transmission electron microscopy (TEM) revealing the presence of electron-dense calciumphosphate accumulations (Fig. 4d), analogous to the formation of hydroxyapatite during amelogenesis [53]. This mineralization outcome appeared even more prominent when the MIM-cultured organoids were incubated in an in vivo environment, i.e. following subcutaneous transplantation of 3D-printed hydroxyapatite scaffolds seeded with organoids in immunodeficient mice (Supplementary Fig. 4c; ARS and Masson's trichrome staining (TCM)).

Finally, to validate whether the ameloblast differentiation capacity is already present in the organoid-initiating ERM stem cells, we developed the organoids immediately in MIM and subsequently analyzed their phenotype (in P1; Supplementary Fig. 4d). ODAM expression was detected in the MIM- (but not TOM-) grown organoids coinciding with the almost absence of SOX $2^{+}$cells. AMTN and KLK4 were also detected at higher levels in MIM- versus TOMgrown organoids, while MMP20 expression was only lowly expressed by both organoid types (Supplementary Fig. 4d).

Taken all together, our new tooth organoid model is capable of unfolding an ameloblast differentiation process involving known consecutive steps, thereby recapitulating DESC/ ERM functionality, and thus provides a valuable research tool to study amelogenesis of human tooth, at present poorly defined.

\section{Single-cell transcriptomics of tooth organoids enriches insights into amelogenesis}

To decipher the amelogenesis differentiation process that occurs in the tooth organoids in deeper detail, we performed scRNA-seq analysis of P4 organoids switched to MIM for 8 days (referred to as P4-switch; see Fig. 4a), and integrated the data with the scRNA-seq dataset described above (Fig. 5a).

As expected, stemness markers (e.g. SOX2, KRT15) are more prominent in the non-differentiated $\mathrm{P} 4$ organoid cluster, whereas ameloblast differentiation markers (e.g. AMTN, $O D A M)$ show almost exclusive expression in the differentiated P4-switch organoids (Fig. 5b), all concordant with 
(a) $\mathrm{P5}$
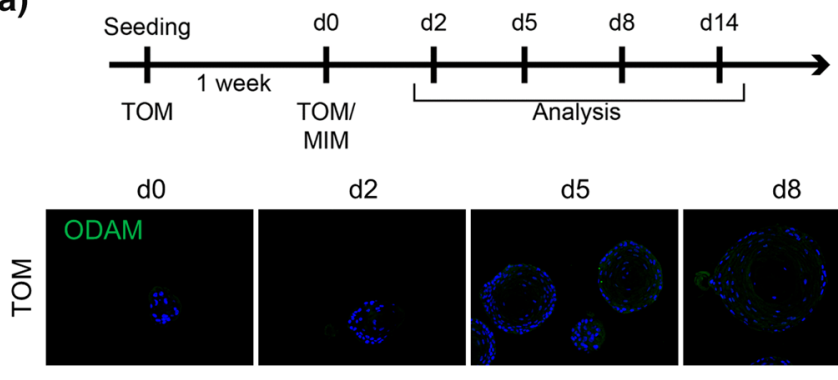

d2
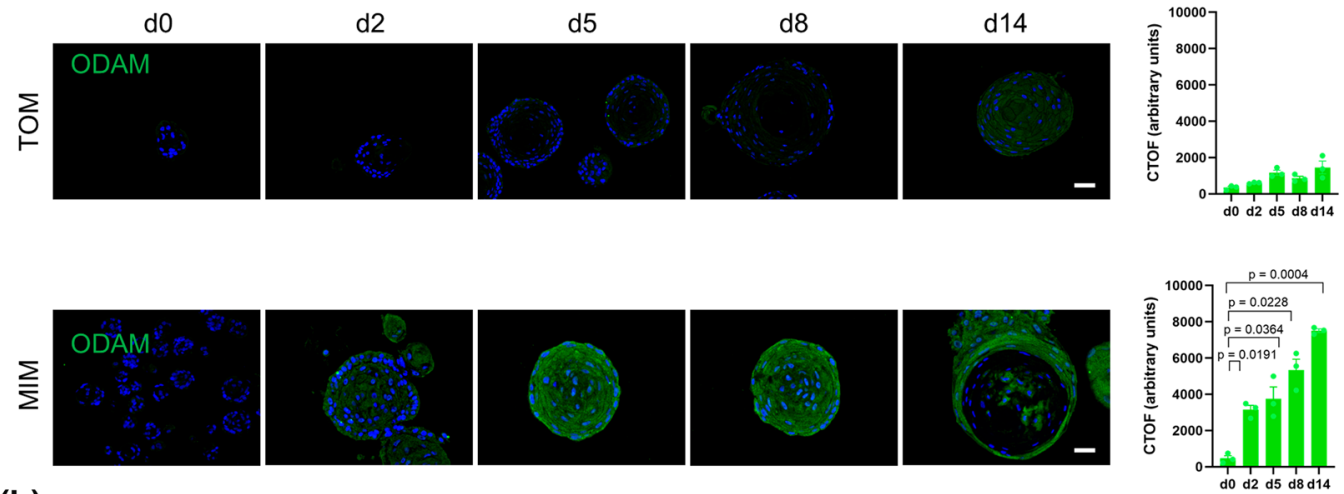

(b)
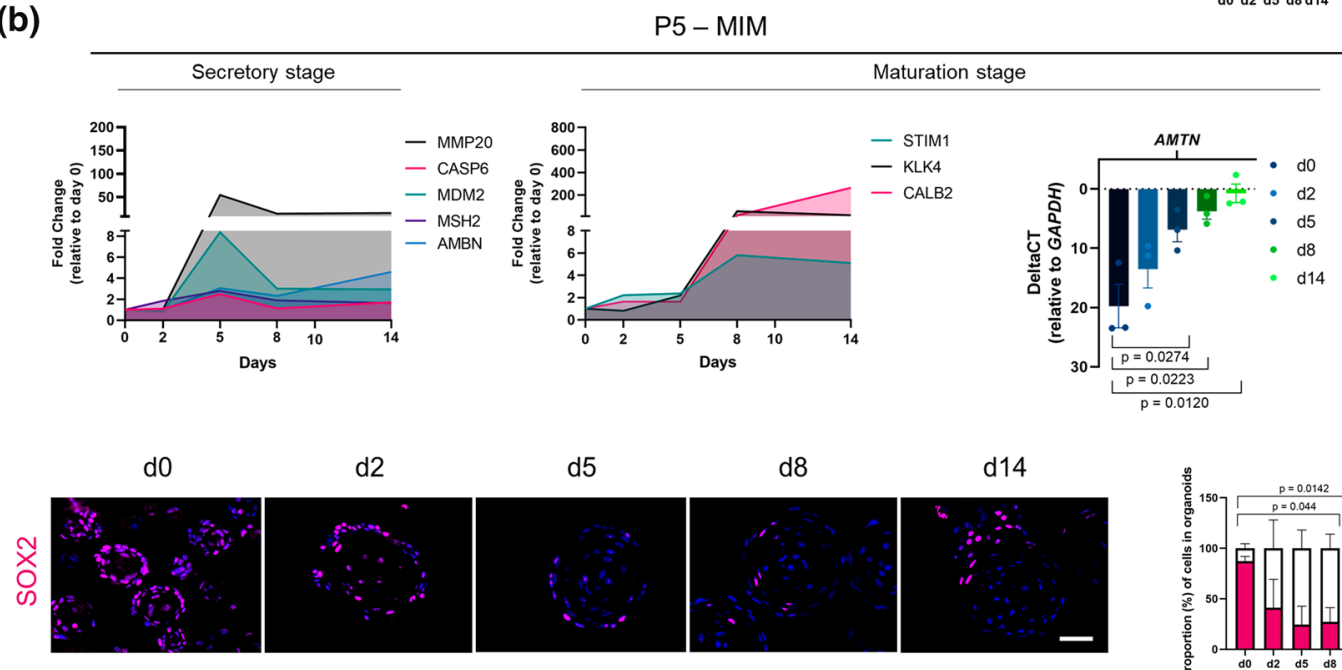

d2

d5

d8
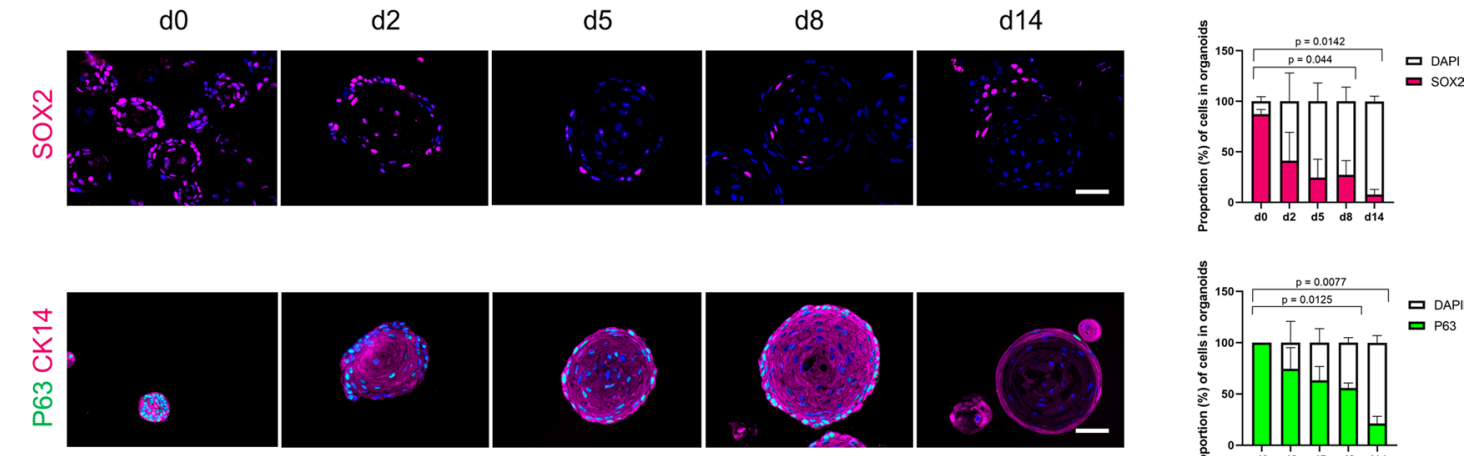

(c)
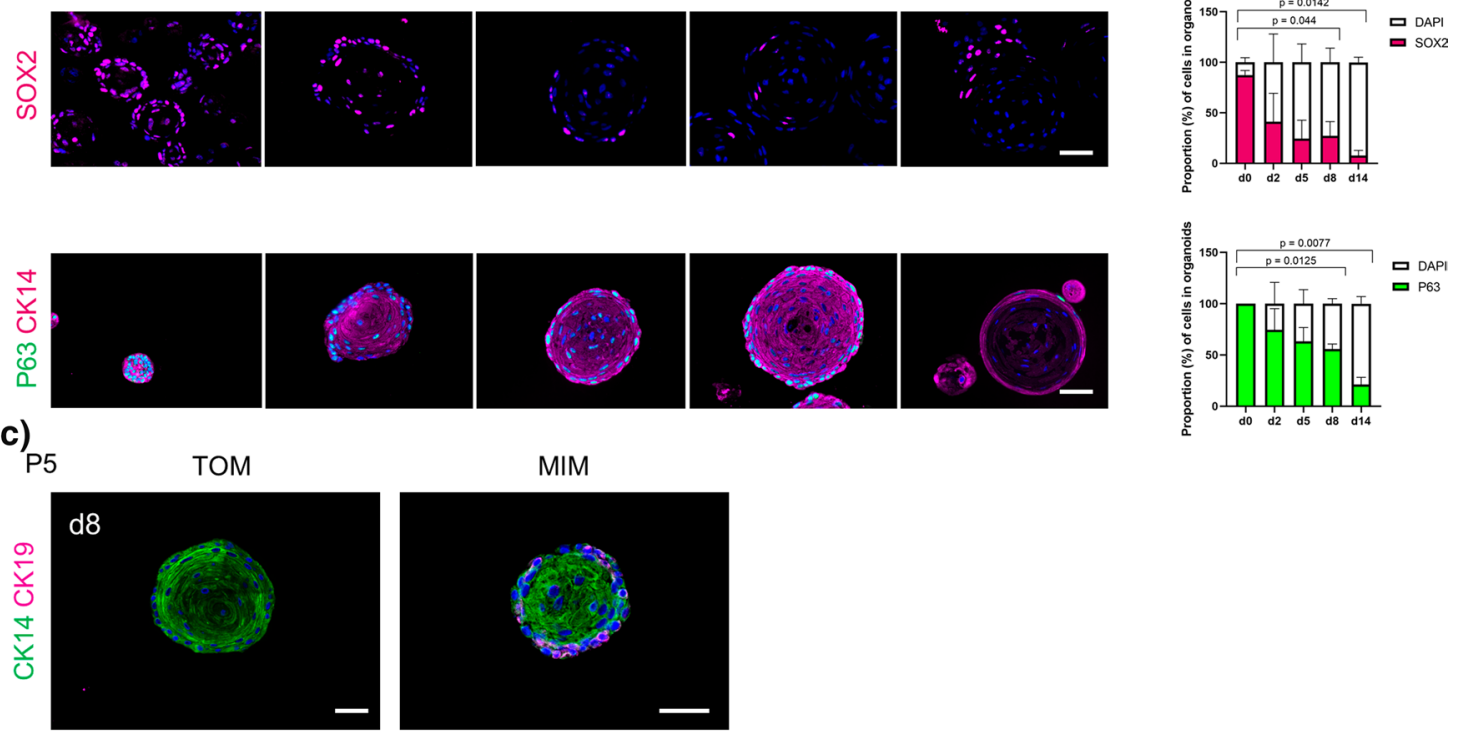

(d)
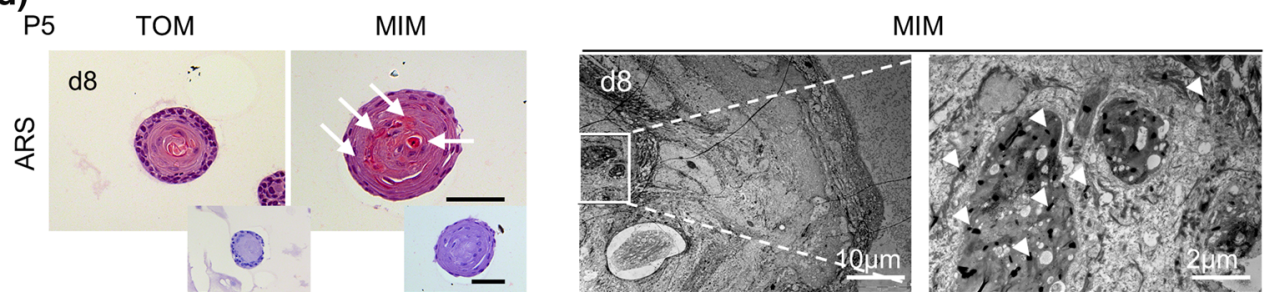
4Fig. 4 Ameloblast differentiation-mimicking process in tooth organoids. a Timeline of experimental set-up (d, day). Immunofluorescence examination of ODAM in organoids from culture conditions and time points as indicated. DAPI (blue) was used to label nuclei. Corrected total organoid fluorescence (CTOF) quantification of ODAM in organoids at indicated time points (mean \pm SEM; $n=3$ biological replicates). b Gene expression pattern of ameloblast secretory- and maturation-stage markers in MIM-switched organoids at time points as indicated. Data are expressed as fold change relative to the organoids at switching to MIM (d0). Expression is normalized to expression of $G A P D H$. Data are mean of $n=3$ biological replicates. Right: Gene expression levels (relative to GAPDH) of AMTN in MIM-switched organoids at time points as indicated (mean \pm SEM; $n=3$ biological replicates). Below: Immunofluorescence staining for the indicated markers, and quantification of $\mathrm{SOX}^{+}$and $\mathrm{P}_{3} 3^{+}$cells in organoids cultured in MIM (mean \pm SEM; $n=3$ biological replicates). DAPI (blue) was used to label nuclei. c Immunofluorescence staining for the indicated markers in organoids cultured as specified. DAPI (blue) was used to label nuclei. d Alizarin Red S (ARS) staining of organoids cultured as specified. Arrows indicate $\mathrm{ARS}^{+}$areas. Images below show negative control (i.e. hematoxylin only). Right: ultrastructural (TEM) analysis of MIM-switched organoids. Boxed area is enlarged. Arrowheads indicate calcium phosphate crystals. Scale bars: $50 \mu \mathrm{m}$, unless indicated otherwise

the findings above. Analogously, the newly identified EGRI and ATF3 are mainly expressed in the non-differentiated P4 organoid cluster, while the tooth development marker PITX2 was found in both stemness and differentiated organoid groups (Supplementary Fig. 5a).

Looking more broadly at gene expression differences using DEG analysis (Supplementary Dataset 6) revealed that P4 and P4-switch organoid clusters clearly display different gene signatures, thereby exposing interesting (new) markers (Fig. 5c). Among others, ornithine decarboxylase 1 (ODC1), a gene involved in cell cycle regulation [58] and proposed as a marker of dental epithelium (moreover aberrantly expressed in specific odontogenic tumors [58]), is higher expressed in $\mathrm{P} 4$ versus $\mathrm{P} 4$-switch organoids (Fig. 5c). In addition to KRT15 and ATF3 belonging to the top 10 upregulated DEGs in P4 organoids (Fig. 5c), other undifferentiated epithelial cell markers are also distinctly expressed in P4 versus P4-switch organoids, including death-associated protein-like 1 (DAPL1) and tissue inhibitor of metalloproteinases 1 (TIMP1) (Fig. 5c), both recently discovered markers of mouse dental epithelium [46, 59]. On the other hand, in addition to AMTN and ODAM surfacing in the top $10 \mathrm{DEGs}$ of P4-switch as compared to P4 organoids (Fig. 5c; Supplementary Dataset 6), laminin subunit gamma 2 (LAMC2) and laminin subunit alpha 3 ( $L A M A 3)$, both expressed in mature ameloblasts in mouse incisor [46] and essential for amelogenesis in humans (with mutations linked to amelogenesis imperfecta $[60,61])$, are highly upregulated in P4-switch organoids (Fig. 5c), further validated by RT-qPCR (Supplementary Fig. 5b). Follicular dendritic cell secreted peptide
(FDCSP), reported to bind to hydroxyapatite [62], is also distinctly expressed in the P4-switch organoids (Fig. 5c).

GO analysis revealed enriched 'negative regulation of cell differentiation' in the straight $\mathrm{P} 4$ organoids when compared to P4-switch organoids (Supplementary Fig. 5c; Supplementary Dataset 4e, 6). In the reverse comparison, GO analysis exposed enrichment of 'epithelial cell differentiation', 'biomineral tissue development', 'odontogenesis' and 'calcium-mediated signaling' in P4-switch versus P4 organoids (Fig. 5d; Supplementary Dataset 4f). Interestingly, also TGF $\beta$-associated processes are upregulated (Fig. $5 \mathrm{~d}$ ), in line with the enrichment of 'negative regulation of SMAD protein signal transduction' in the non-differentiated organoids (Supplementary Fig. 5c), and the knowledge that the TGF $\beta$ pathway plays an important role in ameloblast differentiation [63].

Next, we performed gene set enrichment analysis (GSEA) [64] which exposed several important differentiation (amelogenesis) pathways in P4-switch versus P4 organoids. Firstly, mineralization hallmarks (tooth, enamel) are significantly enriched in P4-switch organoids (Fig. 5e). In addition, calcium-signaling pathways, highly important during amelogenesis [53], were found significantly associated with the P4-switch organoids such as the hallmarks 'calmodulin binding', 'store-operated calcium entry' and 'calcium mediated signaling' (Supplementary Fig. 5d). Further interestingly, GSEA revealed significant enrichment of TGF $\beta$ signaling hallmarks in $\mathrm{P} 4$-switch versus $\mathrm{P} 4$ organoids, more specifically TGF $\beta$ (receptor) signaling and TGF $\beta$ (particularly TGF $\beta 1 / 3$ ) production (Fig. 5e), in line with the importance of the TGF $\beta$ pathway in amelogenesis [63].

Regulon analysis exposed higher activity of the signal transducer and activator of transcription 2 (STAT2) generegulatory network in $\mathrm{P} 4$-switch than $\mathrm{P} 4$ organoids (Fig. 5f). STAT2 is specifically found in ameloblasts (reported in neonatal rat molars [65]) and positively targets $A M T N$, the ameloblast-related $L A M C 2$ and $L A M B 3$, as well as genes associated with TGF $\beta$ signaling ( $T G F \beta 3, T G F \beta R 2)$ (Fig. 5f). Also, avian musculoaponeurotic fibrosarcoma (MAF), specifically expressed in ameloblasts (reported in mouse incisor tooth germs [66]) and representing an essential regulator of AMELX secretion during amelogenesis [66], shows higher regulon activity in P4-switch than P4 organoids (Fig. 5f). MAF is predicted to positively regulate fibronectin $(F N 1)$ and $R U N X 2$, genes related to ameloblast differentiation, and TGF $\beta$ signaling-associated SMAD3, $S M A D 6$, and TGF $\beta$-induced (TGF $\beta I$ ), an activated form of the TGF $\beta 1$ ligand (Fig. 5f). RUNX2 expression has been reported in ameloblasts during the late secretory and maturation stages and its deletion suppresses enamel maturation [67]. In addition, TGF $\beta 1$ affects enamel mineralization by modulating RUNX2 [67, 68]. TGF $\beta I$ is also an important 
predicted target gene of Forkhead Box C2 (FOXC2), and FOXC2 regulon activity was found higher in $\mathrm{P} 4$-switch than $\mathrm{P} 4$ organoids (Fig. 5f). FOXC2 is highly expressed during craniofacial development [69], but its exact role during tooth development and differentiation is unknown. FOXC2 is also predicted to positively regulate $L A M C 2$ and $M S X 1$, a highly conserved transcription factor well-known to regulate tooth formation [4], and causing tooth agenesis in humans when mutated [70]. Finally, SOX4 and HMGA2 regulons are prominently activated in $\mathrm{P} 4$-switch organoids (Supplementary Fig. 5e). SOX4 expression has been reported in DESCs and in inner enamel epithelium (at the cap stage in mouse) [71] and targets PITX2, while HMGA2 is involved in early tooth formation and stem cell marker (e.g. SOX2) expression (a)

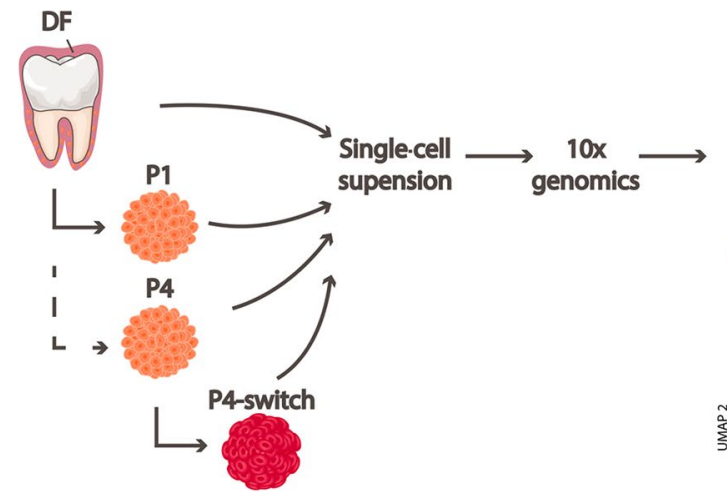

(b) sox2
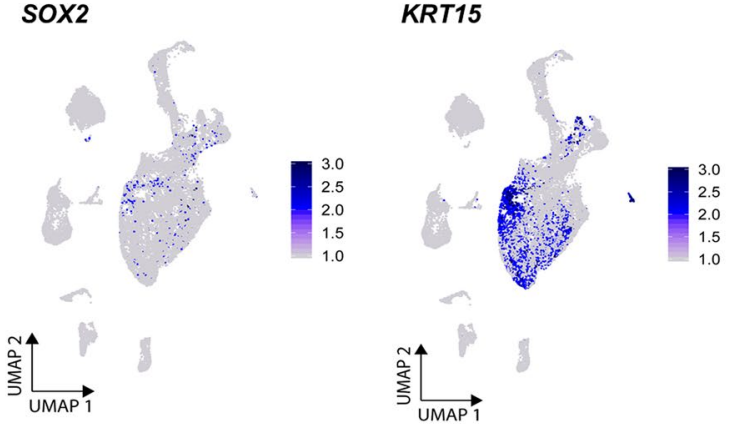

(c) P4

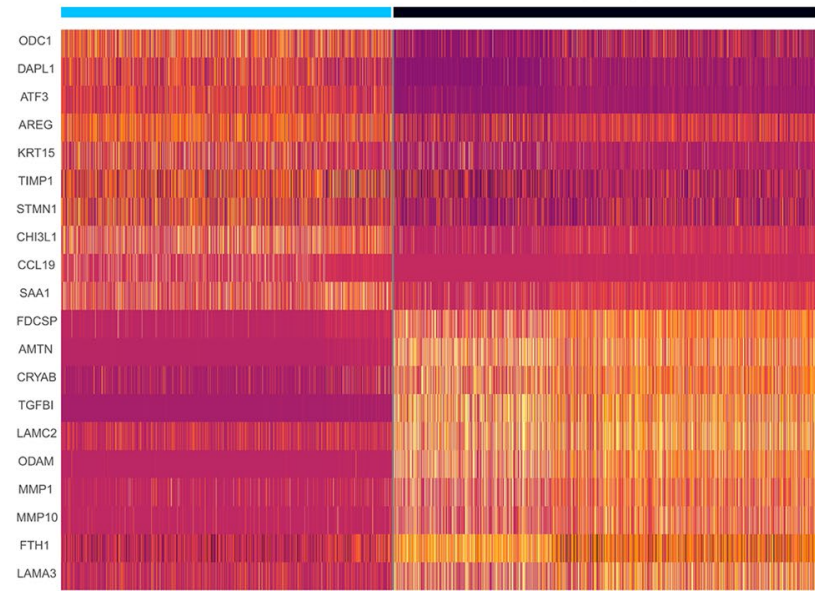

Fig. 5 Single-cell transcriptomic profiling of tooth organoids driven into amelogenesis-resembling differentiation. a Experimental overview of the scRNA-seq analysis. UMAP plot of the integrated DF and organoid samples as indicated. 'Primary' means all DF clusters. b Projection of indicated genes on the integrated UMAP plot. c Heatmap displaying the scaled expression of the top 10 DEGs per cluster in P4 versus P4-switch organoids. d Significant (FDR $\leq 0.05)$

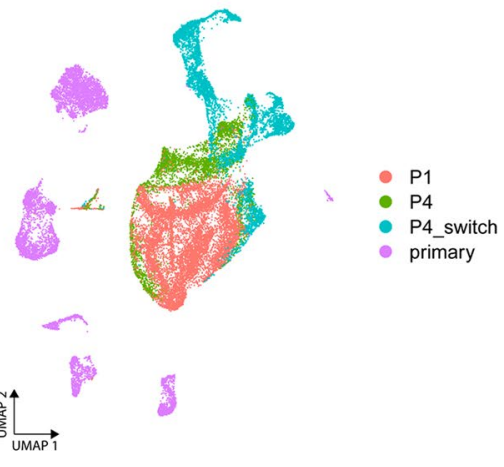

$A M T N$

ODAM
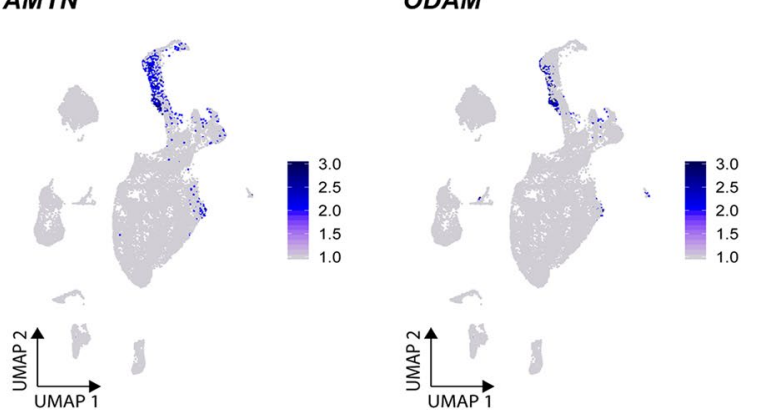

(d)

GO Analysis

P4-switch vs P4

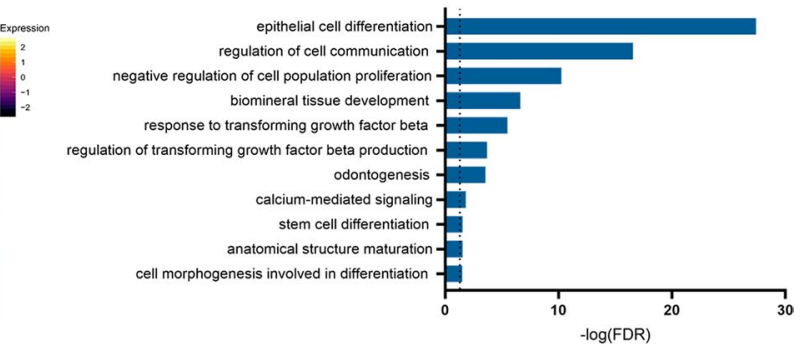

DEG-based GO terms enriched in P4-switch versus P4 organoids. e DEG-based GSEA plots of the indicated hallmarks in P4-switch versus P4 organoids. Normalized enrichment score (NES-), and p- and FDR-values are listed. f Indicated regulons (STAT2, MAF, FOXC2) projected on the integrated UMAP plot. Dot plot of predicted STAT2 or MAF regulon target genes in $\mathrm{P} 4$ and $\mathrm{P} 4$-switch organoids. Projection of $T G F \beta I$ gene expression on the UMAP plot 
(e)
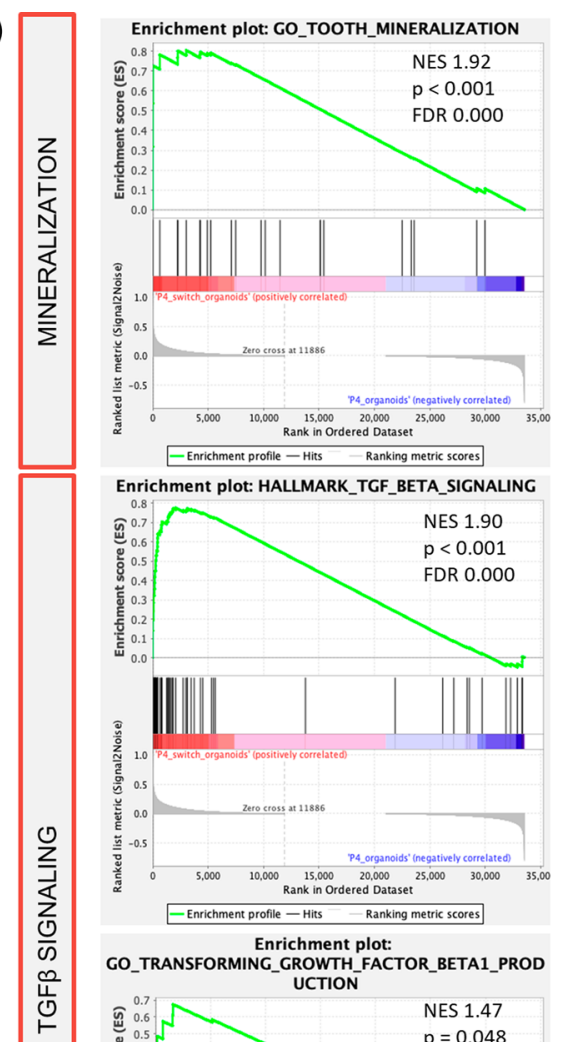

richment plot: HALMMARK TCF BETA SIGNALING

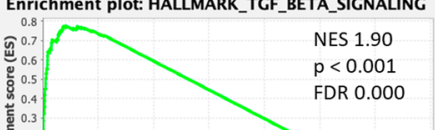

竞

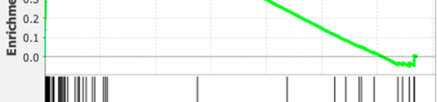

害。

(1)

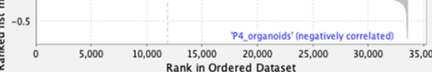

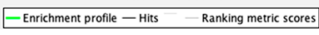

Enrichment plot:

GO_TRANSFORMING_GROWTH_FACTOR_BETA1_PROD

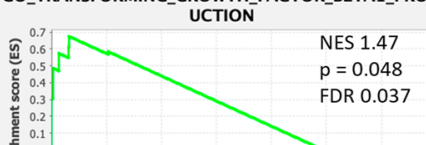

(a)

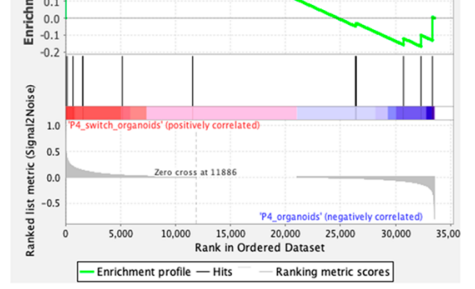

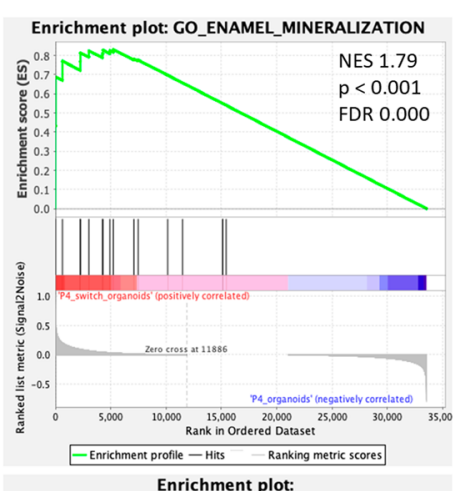

Enrichment plot:
GO_TRANSFORMING_GROWTH_FACTOR_BETA_RECEPT OR_SIGNALING_PATHWAY

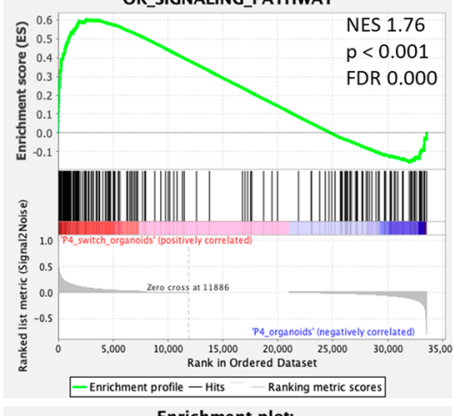

Enrichment plot:
GO_TRANSFORMING_GROWTH_FACTOR_BETA3_PROD
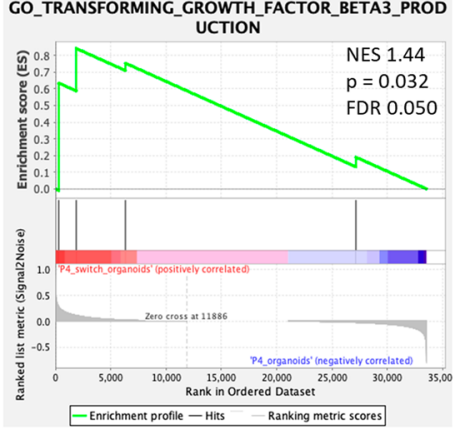

Enrichment plot:
GO_TRANSFORMING_GROWTH_FACTOR_BETA_PRODU

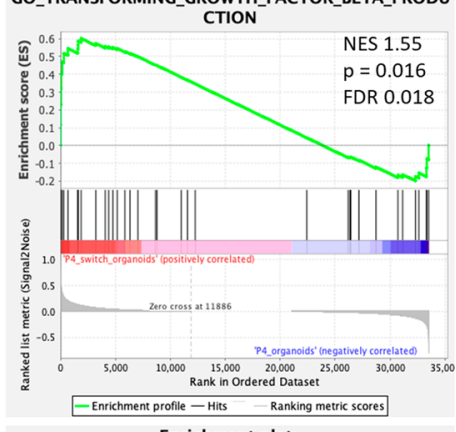

Enrichment plot:
GO_TRANSFORMING_GROWTH_FACTOR_BETA_ACTIVA

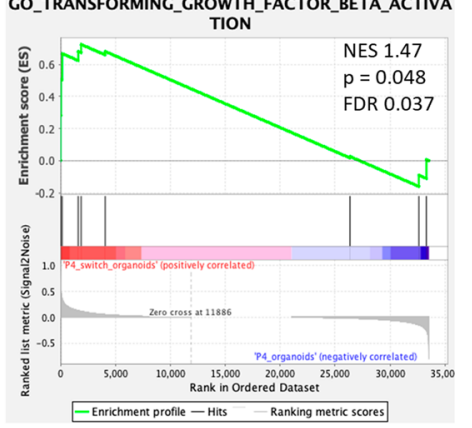

(f)

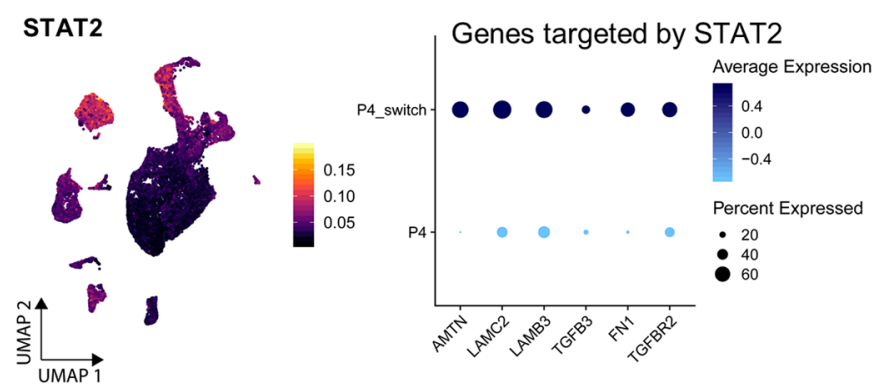

MAF

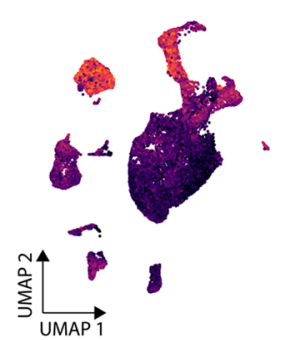

Genes targeted by MAF

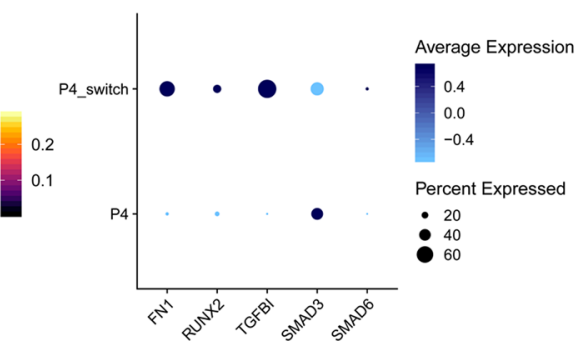

TGFBI

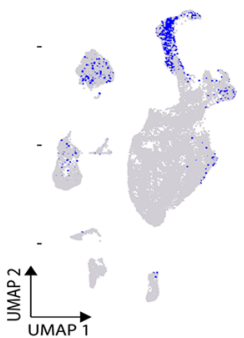

FOXC2

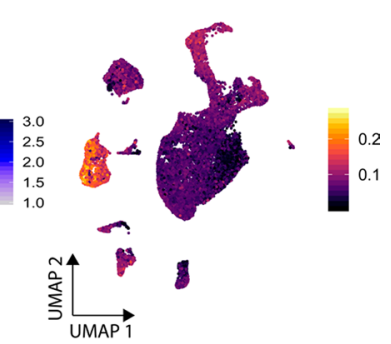

Fig. 5 (continued) 
(a) P5
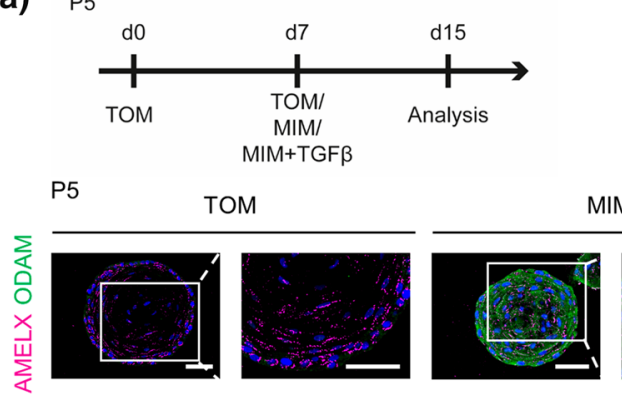

MIM
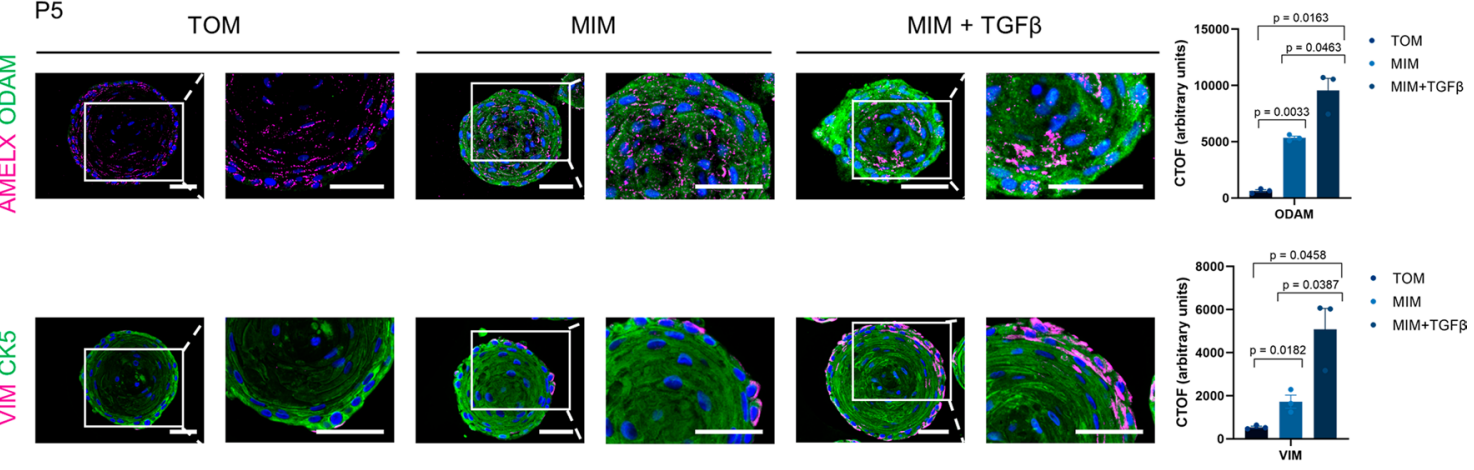

(b)

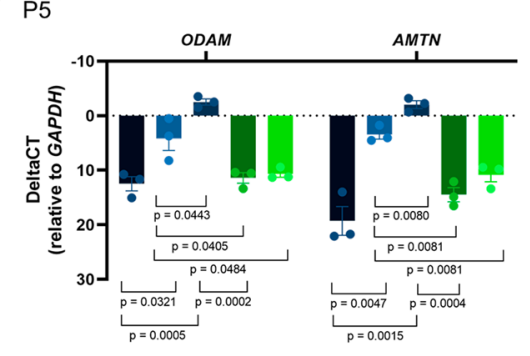

(c)

- TOM

- MIM

- MIM+TGF $\beta+T G F \beta i n h$

MIM+TGF $\beta$ inh

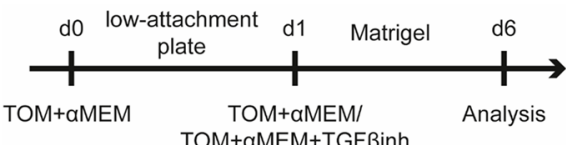

(d)

TOM+aMEM

TOM+aMEM+TGFßinh
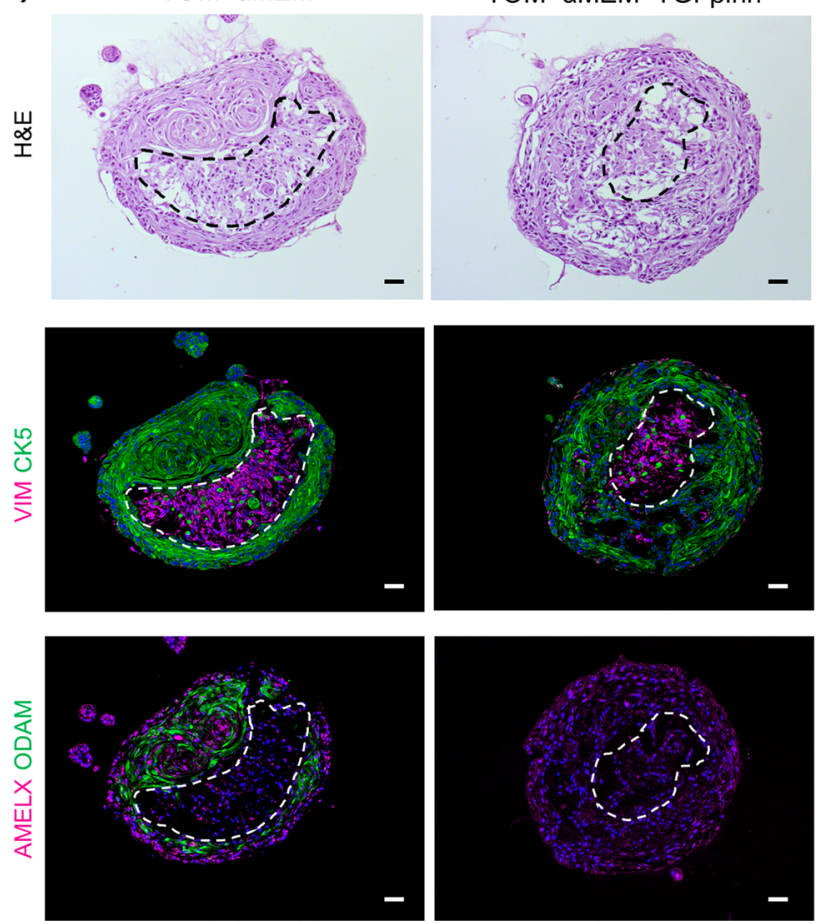
4Fig. 6 Effect of TGF $\beta$ on differentiation in tooth organoids. a Timeline of experimental set-up (d, day). Immunofluorescence examination for the indicated markers in organoids cultured as denoted. Boxed areas are enlarged. DAPI (blue) was used to label the nuclei. CTOF quantification of indicated markers in organoids cultured as specified (mean \pm SEM; $n=3$ biological replicates). b-c Gene expression levels (relative to GAPDH) of indicated markers in organoids cultured as denoted (mean $\pm \mathrm{SEM} ; n \geq 3$ biological replicates). d Timeline of experimental set-up. Histological (H\&E) analysis and immunofluorescence examination for the indicated markers in assembloids cultured as indicated. DAPI (blue) was used to label the nuclei. Dotted area demarcates the $\left(\mathrm{VIM}^{+}\right)$mesenchymal cells. Scale bars: $50 \mu \mathrm{m}$

[72] (hence, plausibly associated with enlarged and supernumerary teeth when truncated [72]), and predicted targets include FN1 and LAMA3 (Supplementary Fig. 5e).

Pseudotime trajectory analysis (using Monocle3 [73]) projected a potential developmental path from P1-P4 to P4-switch clusters (Supplementary Fig. 5f). Intriguingly, the trajectory passes through a particular subcluster of the P4-switch organoids (Supplementary Fig. 5f, encircled), likely representing a transitional stage as supported by the concurrent expression of stemness/development markers (SOX2, KRT15, PITX2) and differentiation markers (AMTN, ODAM) in this subcluster (see Fig. 5b; Supplementary Fig. 5a). Interestingly, regulons controlling ameloblast differentiation (PITX1, DLX3, MEIS1) are especially active in this subcluster (Supplementary Fig. 5g). PITX1 is required for proper tooth formation [74], and has been described in secretory stage ameloblasts [75]. DLX3 promotes the expression of EMPs during amelogenesis [76], and MEIS1 has been shown to bind to DLX3 [77].

In a final analysis of the scRNA-seq data, we applied STRING to in silico predict protein-protein interactions [78]. Using the top 40 DEGs in P4-switch versus P4 organoid clusters, it is projected that AMTN and ODAM closely interact (Supplementary Fig. 5h; Supplementary Dataset 6, 7a), in agreement with former reports [76]. Interestingly, AMTN is also predicted to cooperate with C4orf26, an ECM acidic phosphoprotein suggested to play a key role in enamel mineralization and crystal nucleation [76]. In addition, the STRING analysis proposed interaction of AMTN with LAMB3 suggesting a role in cell-matrix attachment, in line with previously proposed interactions of AMTN with laminins localized in the epithelial basement membrane of the ECM [76]. In the predicted network, LAMB3 interacts with LAMA3 and LAMC2 which is consistent with previous reports [76]. Interestingly, AMTN is also proposed to network with FN1, at present not reported. Moreover, FN1 is predicted to interact with TGF $\beta 1$ and ITG $\beta 6$. ITG $\beta 6$ is known to activate TGF $\beta 1$ by binding to arginine-glycineaspartic acid (RGD) motifs present in ECM proteins such as FN1 [76]. GO-Biological Process analysis of these particular top 40 DEGs in P4-switch organoids confirmed key features of biomineral tissue development, odontogenesis and enamel mineralization, as well as of TGF $\beta$ signaling, the latter further stressed by KEGG pathway analysis (Supplementary Fig. 5i; Supplementary Dataset 7b,c).

Taken together, single-cell transcriptomics of the tooth organoids driven into amelogenesis differentiation demonstrates and underscores the relevance of our new organoid model by confirming known data as well as presenting new insights in the amelogenesis process in humans which is at present far from clarified.

\section{TGF $\beta$ fortifies amelogenesis in tooth organoids and triggers PDL-like differentiation}

The above analyses exposed the enrichment of TGF $\beta$ pathway processes in tooth organoids subjected to ameloblast differentiation, in line with the proposed key role of TGF $\beta$ in amelogenesis [63]. To assess the impact of the TGF $\beta$ pathway, we switched organoids grown in TOM (P5) to MIM with or without TGF $\beta$ (Fig. 6a). Immunofluorescence analysis revealed that addition of TGF $\beta$ further upregulated the expression of ODAM (Fig. 6a), supported by gene expression interrogation also showing increased expression of AMTN (Fig. 6b). The effect of TGF $\beta$ was blocked by the simultaneous addition of a TGF $\beta$ receptor inhibitor (LY2109761, further referred to as TGF $\beta$ inh), thereby demonstrating the specificity of the effect observed (Fig. 6b). Intriguingly, adding TGF $\beta$ inh to MIM-cultured organoids (i.e. without adding exogenous TGF $\beta$ ) strongly reduced the upregulated $O D A M$ and $A M T N$ expression in MIM (Fig. 6b), indicating the presence and implication of endogenous TGF $\beta$ signaling in the differentiation process, in line with our findings above (Fig. 5d,e) and corroborated by the increase in expression of TGF $\beta 1$ and its receptors $T G F \beta R 1$ and $T G F \beta R 2$ in MIM culture (Supplementary Fig. 6a). Together, the data demonstrate that TGF $\beta$ further advances the amelogenesis-mimicking differentiation in the tooth organoids.

During tooth root development, Hertwig's epithelial root sheath (HERS), from which ERM is eventually derived [4, 5], undergoes EMT to develop to participate in PDL formation [47]. It has previously been proposed that this EMT process is regulated by TGF $\beta$, thereby triggering HERS/ERM cells to switch phenotype toward PDL cells $[5,47,79]$. Addition of TGF $\beta$ indeed further increased the expression of VIM in the epithelial $\left(\mathrm{CK}^{+}\right)$organoids (Fig. 6a) and also significantly stimulated the expression of the PDL-specific genes periostin (POSTN) and collagen type III alpha 1 chain (COL3A1) [80] (Fig. 6c).

Taken together, TGF $\beta$ coerces the tooth organoids into more pronounced ameloblast differentiation as well as into 
the direction of PDL development. These findings conform to the known activity of TGF $\beta$ in these tooth developmental processes, and thus again corroborate the strength and validity of our new organoid model. Moreover, they provide supportive evidence that the DF-derived organoids replicate the multipotency of dental (HERS/ERM) epithelial stem cells as proposed to unfold in vivo during tooth development and possibly repair $[5,26]$.

\section{The presence of tooth mesenchymal cells triggers ameloblast differentiation in the epithelial organoids}

Given the importance of mesenchyme-epithelium interactions during tooth development including ameloblast differentiation/amelogenesis [4, 54], we addressed the question whether addition of dental mesenchymal cells had an impact on ameloblast differentiation of the epithelial organoids. We opted to use DPSCs to mimic early stages of tooth development in which DPSC-derived odontoblasts are in close contact with ameloblasts [4]. The DPSCs, isolated, grown and characterized using well-defined standard protocols [81-83], were combined with organoid-derived epithelial stem cells in a layered approach [20], thereby forming composite organoids (assembloids) which were cultured in a mixture of TOM and the DPSC growth medium $\alpha$ MEM (Fig. 6d). The hybrid epithelial-mesenchymal composition was confirmed by CK5-VIM immunofluorescence analysis (Fig. 6d), revealing $\mathrm{VIM}^{+}$mesenchymal cells in the inner part and $\mathrm{CK}^{+}$ epithelial cells at the outer zone of the assembloids (Fig. 6d), and by developing assembloids using eGFP-expressing DPSCs (Supplementary Fig. 6b).

Whereas ODAM is not present in the straight (pure) epithelial organoids cultured in TOM (see above and Supplementary Fig. 6c), it is expressed in the assembloids (Fig. 6d). This induction is not due to the addition of $\alpha$ MEM to TOM (Supplementary Fig. 6c). Interestingly, the epithelial cells neighboring the DPSCs express ODAM, whereas the cells at the outside border of the assembloids (thus, not in direct contact with the mesenchymal cells while more exposed to the (stem cell) medium) do not (Fig. 6d). These findings indicate that the presence of (and even more, the close interface with) mesenchymal (stem) cells drives epithelial stem cells into ameloblast differentiation. Addition of TGF $\beta$ inh completely abolished ODAM protein expression in the assembloids (Fig. 6d) and reduced the expression of AMTN (Supplementary Fig. 6d), indicating the involvement of endogenous TGF $\beta$ signaling in the observed effects. TGF $\beta$ pathway components are indeed expressed in the assembloid culture (Supplementary Fig. 6e); the ligand(s) may originate from the epithelial cells (see Supplementary Fig. 6a), further upregulated by the presence of mesenchymal cells, or may be additionally produced by the mesenchymal cells since both dental cell types have been shown to produce TGF $\beta$ $[84,85]$.

Taken together, ameloblast differentiation of epithelial (organoid) stem cells is triggered by the presence of tooth mesenchymal cells involving TGF $\beta$ signaling, thereby corroborating in vivo findings of interactive mesenchymeepithelium importance, and further validating our model as valuable research tool for exploring human tooth (stem cell) biology.

\section{Discussion}

To our knowledge, our study reports the first-in-time development of a long-term expandable epithelial organoid model derived from human dental tissue. The DFderived organoids show a stemness expression profile congruent with the ERM, previously advanced to encompass DESCs [5]. In addition, single-cell transcriptomics uncovered novel molecular features (such as the stemnessassociated hybrid $\mathrm{E} / \mathrm{M}$ nature, new markers and gene-regulatory networks) for the as yet ill-defined and poorly comprehended human DESCs and ERM, interestingly often mirroring very recent findings in mouse $[43,46]$. Noticeably, organoid culturing appeared to proliferatively (re-) activate the ERM stem cells, indeed reported to be highly quiescent in vivo [7, 12]. Moreover, described (stem cellrelated) functional properties of the ERM were markedly recapitulated by the tooth organoids. First, exposure to EGF induced transient proliferation and eventual EMT and migration, thereby mimicking events taking place in the ERM in vivo (for instance, upon tooth insult) [5, 26, 51]. Second, the tooth organoids displayed the capacity to unfold an ameloblast differentiation process, as occurring in vivo during tooth formation [4] and reported for ERM [6, 7, 35, 54], thus recapitulating this ERM differentiation capacity. The organoids displayed molecular changes constituting pathways that underlie ameloblast differentiation during amelogenesis [52, 53]. In addition, the organoids recovered the key position of TGF $\beta$ in ameloblast differentiation/amelogenesis $[63,67,76]$, as well as in PDL development [5, 47, 79, 80]. Moreover, our scRNA-seq interrogation advanced molecular transitions not revealed before in human amelogenesis. Also, STRING analysis projected protein-protein interactions that may further deepen our knowledge on amelogenesis in human tooth, at present not understood. Together, our new model has the potential to in detail decipher ameloblast development and their production of enamel, the quintessential component of our teeth, which would represent an enormous leap forward in the dental field (especially for future dental tissue replacement therapies). Third, the organoid transcriptome reflected functional processes 
before (provisionally) assigned to the ERM [5, 26], including regulation of bone mineralization, osteoblast differentiation and tooth eruption. Hence, our new model may also serve as an interesting tool to help decipher the multiple biological functions assigned to the ERM, at present still debated [5]. Importantly, the organoids show strong expandability, thereby overcoming current hurdles of primary ERM/DESC culturing, such as limited cell number, life span and phenotypical loss. The expansion ability will be highly instrumental for allowing in-depth analysis of this yet enigmatic cell population. Finally, the induction of ameloblast differentiation by the presence of mesenchymal cells, thereby recapitulating the acknowledged importance of epithelium-mesenchyme interaction in tooth development including amelogenesis, again further corroborated the biomimetic value of our new model(s). Altogether, the several characterizations provide strong evidence that our new human tooth (DF)-derived organoid models, to our knowledge not developed before, present a valuable tool to study human tooth epithelial stem-cell biology and development, at present far from understood.

Organoid technology is also highly applicable to human disease modeling in vitro. It has been suggested that ERM cells are associated with the pathogenesis of odontogenic cysts and tumors [5]. Developing organoids from these lesions may help to gain better insight in their pathogenesis. More in general, our tooth organoid approach may be harnessed to model and study tooth diseases ranging from impact of bacteria to genetic mutations (like mutations in P63 and PITX2 [33] associated with tooth anomalies and amelogenesis imperfect $[60,61])$, eventually leading to novel therapeutic targets and treatments.

Finally, organoids have been shown amenable to regenerative replacement therapy [86]. It is tempting to speculate that damaged, lost or missing teeth, causing major health problems [1-3], may in the future be regenerated or replaced by transplanting biological tooth constructs. Such approach may be superior (both material- and function-wise) to the traditional, still suboptimal synthetic implants, among others suffering from lack of physiological functionality, inferior bone integration and absence of innervation. Embryonically derived, bioengineered mouse tooth germs have been shown capable of forming a functional tooth unit after transplantation in an emptied dental cavity of the mouse [18, 20]. Our organoid and assembloid models may provide essential puzzle pieces toward developing human tooth germs. Although transplantation of natural teeth has been performed in some patients, especially children and young adolescents, the availability of such teeth remains limited. Of important note, the murine Matrigel should then be replaced by a clinically compatible ECM mimic. Currently, attempts are being made to substitute Matrigel for defined synthetic hydrogels [87, 88], although achievements are still limited. In conclusion, we developed a long-term expandable stemness organoid model from human tooth, replicating molecular and functional features of the originating epithelial stem cell compartment. The new in vitro model will be highly valuable to explore human tooth epithelial stem cell phenotype and biology such as ameloblast differentiation. Moreover, our study indicates that the postnatal human tooth still contains epithelial stem cells, and the organoids will be beneficial to address the question on their role(s), and on the reasons why they do not, or not prominently, regenerate tooth tissue in postnatal life. This search also implicates the question whether these stem cells can in vivo be re-activated for repair. This understanding may eventually instigate toothregenerative approaches by re-activating endogenous repair capacity and processes.

\section{Material \& methods}

\section{Isolation and dissociation of dental follicle}

Third molars, predominantly unerupted, were extracted from adolescent patients (Supplementary Table 2) at the 'Oral and Maxillo-Facial Surgery_-Imaging \& Pathology (OMFSIMPATH)' unit of University Hospitals (UZ) Leuven after informed consent. The study was approved by the Ethics Committee Research UZ/KU Leuven (13/0104U). For sample collection, the gingiva was pushed aside after which the bone was perforated and the third molars with associated DFs were carefully isolated (without the visually distinct gingiva). DF tissue was diligently peeled from the tooth and collected in Eagle's Minimum Essential Medium ( $\alpha$ MEM; Sigma-Aldrich) supplemented with $10 \%$ fetal bovine serum (FBS; Sigma-Aldrich), 1\% penicillin-streptomycin (Gibco) and $0.5 \%$ fungizone (Amphotericin B; Gibco). Following short rinsing steps in $70 \%$ ethanol and phosphate-buffered saline (PBS; Gibco), tissue was minced into small $\left(\sim 1 \mathrm{~mm}^{2}\right)$ fragments, and further dissociated using collagenase VI ( $3 \mathrm{mg} / \mathrm{ml}$; Thermo Fisher Scientific) and dispase II (4 mg/ $\mathrm{ml}$; Sigma-Aldrich) for $2 \mathrm{~h}$ at $37^{\circ} \mathrm{C}$, while regularly pipetting up and down. The single cells and few small cell clusters were collected through a $40 \mu \mathrm{m}$ cell strainer (Corning) while removing the remaining larger and fibrous tissue fragments.

\section{Establishment and passaging of tooth organoid culture}

The dissociated DF cell material was resuspended in a mixture of serum-free defined medium (SFDM; Thermo Fisher Scientific; Supplementary Table 4 and [89]) and growth factor-reduced Matrigel (Corning) in a 30:70 ratio, which was plated in 48 -well plates at 20,000 cells per $20 \mu \mathrm{L}$ drop. After solidification, tooth organoid medium (TOM; 
Supplementary Table 1), unless indicated otherwise, was supplemented. ROCK inhibitor (RI; $10 \mu \mathrm{M}$; Merck Millipore) was added the first day of seeding (or passaging). Organoid cultures were kept at $37{ }^{\circ} \mathrm{C}$ in a $1.9 \% \mathrm{CO}_{2}$ incubator, and medium was refreshed every 2-3 days, each time supplemented with fungizone $(0.1 \%)$.

The organoid cultures were passaged every 10-14 days. Matrigel droplets were collected using ice-cold SFDM, and organoids dissociated using TrypLE (containing $5 \mu \mathrm{M}$ RI; Thermo Fisher Scientific) and mechanical trituration. Remaining large organoid fragments were allowed to sediment and the supernatant, containing single cells and small fragments, seeded as described above. A split ratio of 1:6 was applied once the culture reached stable growth (typically from P2 to P4). Organoids were cryopreserved as previously described $[14,15]$ and stored in liquid nitrogen.

To assess clonal derivation, dissociated single organoid cells were transduced with the lentiviral vector LV-eGFP [90] during $30 \mathrm{~min}$ at $37^{\circ} \mathrm{C}$, resulting in $60 \% \mathrm{eGFP}^{+}$cells as analyzed by flow cytometry. The resulting mixture of $\mathrm{eGFP}^{+}$ and $\mathrm{eGFP}^{-}$cells was seeded in organoid culture as described above, and cultures analyzed 14 days later using brightfield and epifluorescence microscopy (Axiovert 40 CFL; Zeiss).

\section{FACS isolation of ITGa $6^{ \pm}$cells from DF}

Primary DFs were dissociated into single cells as described above. Cells were incubated with PE-anti-ITG $\alpha 6$ antibody (1:5; Cat.no 555736; BD Biosciences) and rinsed, both performed in TOM supplemented with fungizone $(0.1 \%)$ and RI $(10 \mu \mathrm{M})$. ITG $\alpha 6^{+}$and ITG $\alpha 6^{-}$cells within the living (DAPI-negative) population were sorted in TOM (supplemented with fungizone and RI) using a BD Influx (BD Biosciences), and seeded at 7500 cells per $20 \mu \mathrm{L}$ Matrigel droplet as mentioned above. RI $(10 \mu \mathrm{M})$ was added to the cultures for 1 week.

\section{In vitro differentiation of the DF-derived epithelial organoids}

Organoids (or dissociated DF) were cultured in mineralization-inducing medium (MIM; Supplementary Table 3; time schedule, see Fig. 4a and Supplementary Fig. 4d) as described above. Recombinant human TGF $\beta 1$ (10 ng/ $\mathrm{ml}$; R\&D) and the selective TGF $\beta$ receptor $1 / 2$ inhibitor LY2109761 (5 $\mu \mathrm{M}$; Selleckchem) were added when indicated.

\section{In vivo transplantation of the DF-derived epithelial organoids}

Matrigel $(10 \mu \mathrm{L})$ with dissociated organoid cells $(150,000)$ was pipetted into custom-made 3D-printed hydroxyapatite constructs (Sirris) which were subcutaneously transplanted in immunodeficient nu/nu mice (Janvier Labs), as in detail described elsewhere [91]. After 4 weeks, implants were resected and subjected to $48 \mathrm{~h}$-fixation in $4 \%$ paraformaldehyde (PFA) (Sigma-Aldrich), paraffin-embedding, 24 h-decalcification, 7- $\mu \mathrm{m}$ sectioning and Alizarin Red S (ARS) or Masson's Trichrome (TCM) staining as described [91]. The study was approved by the Ethical Committee on Animal Experiments (ECAE) of Hasselt University (proto$\operatorname{col} 202,044)$.

\section{Dental pulp stem cell culture}

DPSCs were obtained as in detail described and characterized before [81-83]. In short, dental pulp was collected from the extracted wisdom teeth (after careful removal of the apical papilla), minced and fragments cultured in T25 flasks (Corning) in $\alpha$ MEM supplemented with $10 \%$ fetal bovine serum (FBS) and 1\% L-glutamine (Gibco). When 70-80\% confluence was reached, cells were trypsinized and re-plated at 150,000 cells per T75 flask, and used at early passage ( P5) for assembloid creation. For GFP labelling, DPSCs were transduced with the lentiviral vector LV-eGFP [90] as described above.

\section{Development and culture of assembloids}

Organoid and DPSC cultures were dissociated into single cells, and mixed in a round-bottom low-attachment plate (96-well; Greiner) using a layered approach [20]. First, DPSCs $\left(5 \times 10^{4}\right.$ cells) were sedimented by centrifugation (300 $\mathrm{g}$ for $1 \mathrm{~min}$ at $4{ }^{\circ} \mathrm{C}$ ), followed by deposition of the organoid-derived cells $\left(1 \times 10^{5}\right.$; at $300 \mathrm{~g}$ and $4{ }^{\circ} \mathrm{C}$ for $\left.2 \mathrm{~min}\right)$. The cells were layered in 10\% Matrigel and $90 \%$ of a 1:1 mixture of TOM (i.e. organoid growth medium) and $\alpha$ MEM (i.e. DPSC growth medium), and then incubated for $24 \mathrm{~h}$ at $37{ }^{\circ} \mathrm{C}$ in $5 \% \mathrm{CO}_{2}$. The formed aggregate was re-plated into a 48-well plate in a $20 \mu \mathrm{L}$ Matrigel (70\%) droplet as described above to generate the assembloid, further cultured in TOM $+\alpha$ MEM with or without the TGF $\beta$ receptor inhibitor LY2109761 $(5 \mu \mathrm{M})$ as indicated.

\section{Histochemical and immunostaining analysis}

Primary DF tissue, organoids and assembloids were fixed in $4 \%$ PFA for $1 \mathrm{~h}$, embedded in paraffin, and sections subjected to hematoxylin and eosin (H\&E), immunofluorescence or ARS staining. Antigen retrieval (10 mM citrate, pH6) and permeabilization (0.1\% Triton X-100; SigmaAldrich) were performed. After incubation with primary and secondary antibodies (Supplementary Table 5), sections were mounted with Vectashield (DAPI; Vector Laboratories) or DPX mountant (Sigma-Aldrich). Analysis was done using 
a Leica DM5500 epifluorescence microscope or a Zeiss Axioimager epifluorescence microscope. ImageJ software was used to quantify immunoreactive signal intensity and the 'corrected total organoid fluorescence' (CTOF) (=integrated density - (area of organoid $\times$ mean fluorescence of background readings).

\section{Transmission electron microscopy}

Organoid samples were prepared for transmission electron microscopy (TEM) as in detail described before $[15,50]$. In short, samples were fixed in glutaraldehyde/osmium tetroxide, dehydrated, embedded in epoxy resin, and cut into $40-70 \mathrm{~nm}$ sections. TEM analysis was performed with the JEM1400 transmission electron microscope (JEOL) equipped with an Olympus SIS Quesmesa $11 \mathrm{Mpxl}$ camera, or the Philips EM208 S electron microscope (Philips) equipped with the Morada Soft Imaging System camera with corresponding iTEM-FEI software (Olympus SIS).

\section{Gene expression analysis by RT-qPCR}

RNA was extracted from dissociated DF, organoids and assembloids using the GenElute Mammalian Total RNA Miniprep Kit (Sigma-Aldrich) according to the manufacturer's instructions. RNA was reverse-transcribed (RT) using the Superscript III First-Strand Synthesis Supermix (Thermo Fisher Scientific) and the resultant cDNA samples were analyzed with SYBR Green-based quantitative PCR (qPCR) using the StepOnePlus Real-Time PCR System (AB applied biosystems). Forward and reverse primers (Supplementary Table 6) were designed using PrimerBank and PrimerBlast. Glyceraldehyde-3-phosphate dehydrogenase $(G A P D H)$ was included as housekeeping gene. Relative gene expression levels were calculated as $\Delta \mathrm{Ct}(\mathrm{Ct}$ target$-\mathrm{Ct}$ housekeeping gene) and compared to control (see figure legends).

\section{Single-cell RNA-sequencing analysis}

Primary DF tissue from two individual patients (see Supplementary Table 2), and derived organoids at P1 and P4, or switched to MIM (P4-switch), were dissociated into single cells (as described above) and subjected to scRNA-seq analyses using $10 \times$ Genomics, according to manufacturer instructions. Libraries were generated using the Chromium Single Cell 3' v2 Chemistry Library Kit, Gel Bead \& Multiplex kit $(10 \times$ Genomics), and sequenced on NovaSeq6000. After quality control, raw sequencing reads were demultiplexed, aligned to the human reference genome GRCh38 and processed to a matrix representing the UMI's per cell barcode per gene using CellRanger (v3; $10 \times$ Genomics). Downstream analysis was performed in $\mathrm{R}$ (v.3.6.1) using Seurat (v.3.0) [92].
First, data from the primary DF tissue, P1 and P4 organoids were integrated and analyzed, and subsequently data from P4-switch organoids were added for a next analysis (further referred to as Integration 1 and Integration 2 , respectively). Dead cells and potential doublets (i.e. with $<300$ genes or $>10,000$ genes, $>150,000$ unique molecular identifiers (UMI) and $>15 \%$ mitochondrial RNA) were removed (Supplementary Fig. 2b), resulting in a total of 22,317 cells in Integration 1 and 27,851 cells in Integration 2. Integration anchors were identified using the FindIntegrationAnchors function with default parameters and dims $=1: 30$, and data were integrated across all features. Next, expression levels were scaled, centered and subjected to principal component analysis (PCA). The top 30 PCs were selected and used for UMAP dimensionality reduction [42]. Clusters were calculated by the FindClusters function with a resolution set to 2 and 0.1 for Integration 1 and Integration 2, respectively. Differential gene expression was calculated for each cluster using the MAST package (v.1.12.0; Supplementary Dataset 1). All clusters were annotated based on reported DF and ERM markers and on recent scRNA-seq studies of mouse teeth [12, 27, 28, 33, 34, 43, 46]. Marker genes were defined using FindAllMarkers in Seurat.

Gene ontology analysis (GO) of biological processes was done in Panther [44] using significant differentially expressed genes (DEGs; FDR $\leq 0.05$ and $\log F C \geq 0.25$ ). Gene-regulatory networks (regulons) were identified using SCENIC (pySCENIC; v.0.9.15) [45] in Python (v.3.6.9) as described before [89]. In short, co-expression modules were generated and regulons inferred (with default parameters and hg38_refseq-r80__10kb_up_and_down_tss.mc9nr.feather and hg 38_refseq-r80_500bp_up_and_100bp_down_tss. mc9nr.feather motif collections) resulting in a matrix of AUCell values that represent the activity of each regulon in each cell. The AUCell matrix was imported into Seurat and regulons were projected on the integrated UMAP plot.

Gene-set enrichment analysis (GSEA; v.4.1.0) [64, 89] was performed on $\mathrm{P} 4$ and P4-switch organoids using normalized expression data. Gene sets (hallmarks) tested were obtained from the Molecular Signatures Database (MSigDB; v.7.2) $[64,93]$.

To predict protein-protein interactions with STRING (v.11.0) [78], the top 40 DEGs of P4-switch organoids versus $\mathrm{P} 4$ organoids were used. The cluster analysis was subdivided in three colors by kmeans. The minimum required interaction score was set as medium confidence (0.4).

Finally, the pseudotime trajectory was projected onto the integrated UMAP dimensional reduction generated previously with Seurat (P1, P4 and P4-switch organoids) using the Monocle3 (v1.0.0) [73] package's learn_graph and plot_ cells functions. 
Raw sequencing data are available at ArrayExpress (accession number E-MTAB-10596).

\section{Statistical analysis}

Statistical analysis was performed using GraphPad Prism (v.9.0.0). (Un-)paired two-tailed $t$-student test was applied for comparison of 2 groups or two-way analysis of variance (ANOVA) for multiple comparisons followed by Sidak's test for Multiple Comparison. Statistical significance was defined as $P \leq 0.05$.

Supplementary Information The online version contains supplementary material available at https://doi.org/10.1007/s00018-022-04183-8.

Acknowledgements We are grateful to all staff members of the Oral and Maxillofacial Surgery (MKA) of UZ Leuven, as well as the patients, for their invaluable help and contribution to collect freshly extracted third molars. We would also like to thank Dr. Reinhilde Jacobs and Dr. Elisabeth Tijskens for their help with sample collection. We thank Dr. Diether Lambrechts' group for their technical assistance in $10 \times$ Genomics. Computational resources for scRNA-seq analysis were provided by 'Vlaams Supercomputer Centrum' (VSC), managed by the Fund for Scientific Research (FWO)_Flanders (Belgium). We acknowledge the use of the TEM platforms at VIB-KU Leuven, Biomedical Research Institute (BIOMED) UHasselt, and Institute of Development, Aging and Cancer, Tohoku University (Sendai, Japan). We would also like to thank Dr. Ronald Driesen (UHasselt) for help with CTOF analysis, and Dr. Marianne Carlon's group (particularly Liesbeth De Keersmaecker; KU Leuven) for guiding and assisting in lentiviral transduction. We are grateful to Jeanine Santermans and Marc Jans (UHasselt) for their technical assistance in TEM and staining analyses. We also would like to thank Reena Chinnaraj and Vera Dermesrobian (FACS Core, KU Leuven) for their help with FACS experiments.

Author contributions LH designed the concepts and experiments, performed the experiments and the data analysis, interpreted the results, designed the scientific illustrations and wrote the manuscript; FH processed scRNA-seq data, performed regulon, GSEA and pseudotime analysis, and co-discussed certain results; JC processed scRNA-seq data and assisted in bioinformatical analysis; HK performed and cointerpreted TEM analysis; DL co-supervised the scRNA-seq experiments; IL co-designed concepts and experiments, and interpreted TEM and $\mathrm{H} \& \mathrm{E}$ data; $\mathrm{AB}$ co-supervised the project, co-designed concepts and experiments and performed TEM and in vivo experiments; HV designed and supervised the project, co-developed the concepts and experiments, co-analyzed and-interpreted the data and wrote the manuscript. All co-authors critically read and approved the manuscript.

Funding This work was supported by grants from KU Leuven (Research Fund) and Fund for Scientific Research (FWO) Flanders. L.H. is an FWO PhD Fellow (1S84718N).

Availability of data and material (data transparency) The data that support the findings discussed here are available from the corresponding author upon reasonable request.

Code availability (software application or custom code) Not applicable.

\section{Declarations}

Conflicts of interest/Competing interests (include appropriate disclosures) The authors declare no competing financial interests.

Ethics approval (include appropriate approvals or waivers) The study was approved by the Ethics Committee Research UZ/KU Leuven (13/0104U), and the Ethical Committee on Animal Experiments (ECAE) of UHasselt (protocol 202044).

Consent to participate (include appropriate statements) All human participants provided written informed consent for use of their samples prior to participation in the study.

Consent for publication All human participants provided written informed consent for use of their data for publication after study information was given.

Open Access This article is licensed under a Creative Commons Attribution 4.0 International License, which permits use, sharing, adaptation, distribution and reproduction in any medium or format, as long as you give appropriate credit to the original author(s) and the source, provide a link to the Creative Commons licence, and indicate if changes were made. The images or other third party material in this article are included in the article's Creative Commons licence, unless indicated otherwise in a credit line to the material. If material is not included in the article's Creative Commons licence and your intended use is not permitted by statutory regulation or exceeds the permitted use, you will need to obtain permission directly from the copyright holder. To view a copy of this licence, visit http://creativecommons.org/licenses/by/4.0/.

\section{References}

1. Bensley L, VanEenwyk J, Ossiander EM (2011) Peer reviewed: associations of self-reported periodontal disease with metabolic syndrome and number of self-reported chronic conditions. Prev Chronic Dis 8(3):A50

2. Demmer RT, Desvarieux M (2006) Periodontal infections and cardiovascular disease: the heart of the matter. J Am Dent Assoc 137(10 SUPPL.):S14-S20. https://doi.org/10.14219/JADA. ARCHIVE.2006.0402

3. Uwitonze AM et al (2018) Periodontal diseases and adverse pregnancy outcomes: is there a role for vitamin D? J Steroid Biochem Mol Biol 180:65-72. https://doi.org/10.1016/J.JSBMB.2018.01. 010

4. Yu T, Klein OD (2020) Molecular and cellular mechanisms of tooth development, homeostasis and repair. Development (Cambridge) 147(2):dev184754. https://doi.org/10.1242/dev.184754

5. Davis EM (2018) A review of the epithelial cell rests of Malassez on the bicentennial of their description. J Vet Dent 35(4):290-298. https://doi.org/10.1177/0898756418811957

6. Hamamoto Y, Nakajima T, Ozawa H, Uchida T (1996) Production of amelogenin by enamel epithelium of Hertwig's root sheath. Oral Surg Oral Med Oral Pathol Oral Radiol Endod 81(6):703709. https://doi.org/10.1016/s1079-2104(96)80077-1

7. Shinmura Y, Tsuchiya S, Hata KI, Honda MJ (2008) Quiescent epithelial cell rests of Malassez can differentiate into ameloblastlike cells. J Cell Physiol 217(3):728-738. https://doi.org/10.1002/ jcp. 21546

8. Athanassiou-Papaefthymiou M, Papagerakis $\mathrm{P}$, Papagerakis $\mathrm{S}$ (2015) Isolation and characterization of human adult epithelial 
stem cells from the periodontal ligament. J Dent Res 94(11):15911600. https://doi.org/10.1177/0022034515606401

9. Kim G-H et al (2020) Differentiation and establishment of dental epithelial-like stem cells derived from human ESCs and iPSCs. Int J Mol Sci 21(12):1-16. https://doi.org/10.3390/IJMS211243 84

10. Nam H et al (2014) Establishment of Hertwig's epithelial root sheath/ epithelial rests of Malassez cell line from human periodontium. Mol Cells 37(7):562-567. https://doi.org/10.14348/ molcells.2014.0161

11. Nam H et al (2011) Expression profile of the stem cell markers in human Hertwig's epithelial root sheath/epithelial rests of Malassez cells. Mol Cells 31(4):355-360. https://doi.org/10.1007/ s10059-011-0045-3

12. Tsunematsu T et al (2016) Human odontogenic epithelial cells derived from epithelial rests of Malassez possess stem cell properties. Lab Investig 96(10):1063-1075. https://doi.org/10.1038/labin vest. 2016.85

13. Schutgens F, Clevers H (2020) Human organoids: tools for understanding biology and treating diseases. Annu Rev Pathol 15:211-234. https://doi.org/10.1146/ANNUREV-PATHM ECHDIS-012419-032611

14. Boretto $\mathrm{M}$ et al (2017) Development of organoids from mouse and human endometrium showing endometrial epithelium physiology and long-term expandability. Development 144(10):1775-1786. https://doi.org/10.1242/dev.148478

15. Cox B et al (2019) Organoids from pituitary as a novel research model toward pituitary stem cell exploration. J Endocrinol 240(2):287-308. https://doi.org/10.1530/JOE-18-0462

16. Sato $\mathrm{T}$ et al (2009) Single Lgr5 stem cells build crypt-villus structures in vitro without a mesenchymal niche. Nature 459(7244):262-265. https://doi.org/10.1038/nature07935

17. Artegiani B, Clevers H (2018) Use and application of 3D-organoid technology. Hum Mol Genet 27(2):R99-R107. https://doi.org/10. 1093/hmg/ddy 187

18. Gao X, Wu Y, Liao L, Tian W (2021) Oral organoids: progress and challenges. J Dent Res 100(5):454-463. https://doi.org/10. $1177 / 0022034520983808$

19. Binder M, Biggs LC, Kronenberg MS, Schneider P, Thesleff I, Balic A (2020) Novel strategies for expansion of tooth epithelial stem cells and ameloblast generation. Sci Rep 10(1):4063. https:// doi.org/10.1038/s41598-020-60708-w

20. Nakao $\mathrm{K}$ et al (2007) The development of a bioengineered organ germ method. Nat Methods 4(3):227-230. https://doi.org/10.1038/ nmeth1012

21. Duailibi SE, Duailibi MT, Zhang W, Asrican R, Vacanti JP, Yelick PC (2008) Bioengineered dental tissues grown in the rat jaw. J Dent Res 87(8):745-750. https://doi.org/10.1177/1544059108 08700811

22. Young CS, Terada S, Vacanti JP, Honda M, Bartlett JD, Yelick PC (2002) Tissue engineering of complex tooth structures on biodegradable polymer scaffolds. J Dent Res 81(10):695-700. https:// doi.org/10.1177/154405910208101008

23. Jussila M, Thesleff I (2012) Signaling networks regulating tooth organogenesis and regeneration, and the specification of dental mesenchymal and epithelial cell lineages. Cold Spring Harb Perspect Biol 4(4):a008425. https://doi.org/10.1101/CSHPE RSPECT.A008425

24. Oyanagi $T$ et al (2019) Insulin-like growth factor 1 modulates bioengineered tooth morphogenesis. Sci Rep 9(1):368. https:// doi.org/10.1038/S41598-018-36863-6

25. Baker LA, Tiriac H, Clevers H, Tuveson DA (2016) Modeling pancreatic cancer with organoids. Trends Cancer 2(4):176. https:// doi.org/10.1016/J.TRECAN.2016.03.004

26. Consolaro A, Consolaro MFMO (2010) ERM functions, EGF and orthodontic movement or why doesn't orthodontic movement cause alveolodental ankylosis? Dental Press J Orthod 15(2):24 32. https://doi.org/10.1590/S2176-94512010000200003

27. Luan X, Ito Y, Diekwisch TGH (2006) Evolution and development of Hertwig's epithelial root sheath. Dev Dyn 235(5):1167-1180. https://doi.org/10.1002/dvdy.20674

28. Xiong J, Mrozik K, Gronthos S, Bartold PM (2012) Epithelial cell rests of Malassez contain unique stem cell populations capable of undergoing epithelial-mesenchymal transition. Stem Cells Dev 21(11):2012-2025. https://doi.org/10.1089/scd.2011.0471

29. Juuri E et al (2012) Sox $2^{+}$stem cells contribute to all epithelial lineages of the tooth via Sfrp5 ${ }^{+}$progenitors. Dev Cell 23(2):317. https://doi.org/10.1016/J.DEVCEL.2012.05.012

30. Sanz-Navarro M, Delgado I, Torres M, Mustonen T, Michon F, Rice DP (2019) Dental epithelial stem cells express the developmental regulator Meis1. Front Physiol 10:249. https://doi.org/10. 3389/FPHYS.2019.00249

31. Sun $Z$ et al (2016) Sox 2 and Lef- 1 interact with Pitx2 to regulate incisor development and stem cell renewal. Development 143(22):4115-4126. https://doi.org/10.1242/dev.138883

32. Sanz-Navarro $M$ et al (2018) Plasticity within the niche ensures the maintenance of a Sox $2^{+}$stem cell population in the mouse incisor. Development 145(1):dev155929. https://doi.org/10.1242/ dev.155929

33. Fan $\mathrm{Z}$ et al (2019) Novel PITX2 mutations identified in AxenfeldRieger syndrome and the pattern of PITX2-related tooth agenesis. Oral Dis 25(8):2010-2019. https://doi.org/10.1111/odi.13196

34. Haku K et al (2011) Epithelial cell rests of Malassez modulate cell proliferation, differentiation and apoptosis via gap junctional communication under mechanical stretching in vitro. Bull Tokyo Dent Coll 52(4):173-182. https://doi.org/10.2209/tdcpublication. 52.173

35. Fukumoto $\mathrm{S}$ et al (2014) New insights into the functions of enamel matrices in calcified tissues. Jpn Dent Sci Rev 50(2):47-54. https://doi.org/10.1016/j.jdsr.2014.01.001

36. Fong CD, Hammarström L (2000) Expression of amelin and amelogenin in epithelial root sheath remnants of fully formed rat molars. Oral Surg Oral Med Oral Pathol Oral Radiol Endod 90(2):218-223. https://doi.org/10.1067/MOE.2000.107052

37. Janones DS, Massa LF, Arana-Chavez VE (2005) Immunocytochemical examination of the presence of amelogenin during the root development of rat molars. Arch Oral Biol 50(5):527-532. https://doi.org/10.1016/J.ARCHORALBIO.2004.10.004

38. Bansal AK, Shetty DC, Bindal R, Pathak A (2012) Amelogenin: a novel protein with diverse applications in genetic and molecular profiling. J Oral Maxillofac Pathol 16(3):395. https://doi.org/10. 4103/0973-029X.102495

39. Tanimoto K et al (2012) Differential effects of amelogenin on mineralization of cementoblasts and periodontal ligament cells. J Periodontol 83(5):672-679. https://doi.org/10.1902/JOP.2011. 110408

40. Bosshardt DD, Nanci A (2004) Hertwig's epithelial root sheath, enamel matrix proteins, and initiation of cementogenesis in porcine teeth. J Clin Periodontol 31(3):184-192. https://doi.org/10. 1111/J.0303-6979.2004.00473.X

41. Yan Q, Zhang Y, Li W, DenBesten PK (2006) Differentiation of human ameloblast-lineage cells in vitro. Eur J Oral Sci 114(s1):154-158. https://doi.org/10.1111/j.1600-0722.2006. 00304.X

42. L. McInnes, J. Healy, and J. Melville, UMAP: uniform manifold approximation and projection for dimension reduction. $\operatorname{arXiv}, \mathrm{Feb}$. 2018, 2021. http://arxiv.org/abs/1802.03426.

43. Krivanek J et al (2020) Dental cell type atlas reveals stem and differentiated cell types in mouse and human teeth. Nat Commun 11(1):4816. https://doi.org/10.1038/s41467-020-18512-7 
44. Thomas PD et al (2003) PANTHER: a library of protein families and subfamilies indexed by function. Genome Res 13(9):21292141. https://doi.org/10.1101/gr.772403

45. Aibar $\mathrm{S}$ et al (2017) SCENIC: single-cell regulatory network inference and clustering. Nat Methods 14(11):1083-1086. https://doi. org/10.1038/nmeth.4463

46. Chiba $\mathrm{Y}$ et al (2020) Single-cell RNA-sequencing from mouse incisor reveals dental epithelial cell-type specific genes. Front Cell Dev Biol 8:841. https://doi.org/10.3389/fcell.2020.00841

47. Chen J et al (2014) TGF- $\beta 1$ and FGF2 stimulate the epithelialmesenchymal transition of HERS cells through a MEK-dependent mechanism. J Cell Physiol 229(11):1647-1659. https://doi.org/10. $1002 /$ jcp. 24610

48. Zhang S et al (2020) Single-cell transcriptomics identifies divergent developmental lineage trajectories during human pituitary development. Nat Commun 11(1):1-16. https://doi.org/10.1038/ s41467-020-19012-4

49. Dong J et al (2018) Single-cell RNA-seq analysis unveils a prevalent epithelial/mesenchymal hybrid state during mouse organogenesis. Genome Biol 19(1):31. https://doi.org/10.1186/ s13059-018-1416-2

50. Lambrichts I, Creemers J, Van Steenberghe D (1993) Periodontal neural endings intimately relate to epithelial rests of Malassez in humans: a light and electron microscope study. J Anat 182(2):153-162

51. Guajardo G et al (2000) Immunohistochemical localization of epidermal growth factor in cat paradental tissues during tooth movement. Am J Orthod Dentofac Orthop 118(2):210-219. https://doi. org/10.1067/MOD.2000.104097

52. Liu C et al (2015) Cell cycle control, DNA damage repair, and apoptosis-related pathways control pre-ameloblasts differentiation during tooth development. BMC Genomics 16(1):592. https://doi. org/10.1186/s12864-015-1783-y

53. Nurbaeva MK, Eckstein M, Feske S, Lacruz RS (2017) Ca2+ transport and signalling in enamel cells. J Physiol 595(10):30153039. https://doi.org/10.1113/JP272775

54. Takahashi K, Shimonishi M, Wang R, Watanabe H, Kikuchi M (2012) Epithelial-mesenchymal interactions induce enamel matrix proteins and proteases in the epithelial cells of the rests of Malassez in vitro. Eur J Oral Sci 120(6):475-483. https://doi.org/ 10.1111/J.1600-0722.2012.01002.X

55. Chen J, Zhang Y, Mendoza O, Denbesten P (2009) Calcium-mediated differentiation of ameloblast lineage cells in vitro. J Exp Zool Part B Mol Dev Evol 312(5):458-464. https://doi.org/10.1002/ jez.b.21279

56. DenBesten PK, Machule D, Zhang Y, Yan Q, Li W (2005) Characterization of human primary enamel organ epithelial cells in vitro. Arch Oral Biol 50(8):689-694. https://doi.org/10.1016/j.archoralbio.2004.12.008

57. Crivelini MM, De Araújo VC, De Sousa SOM, De Araújo NS (2003) Cytokeratins in epithelia of odontogenic neoplasms. Oral Dis 9(1):1-6. https://doi.org/10.1034/j.1601-0825.2003.00861.x

58. Heikinheimo K et al (2015) Early dental epithelial transcription factors distinguish ameloblastoma from keratocystic odontogenic tumor. J Dent Res 94(1):101-111. https://doi.org/10.1177/00220 34514556815

59. Yoshiba $\mathrm{N}$ et al (2003) Temporospatial gene expression and protein localization of matrix metalloproteinases and their inhibitors during mouse molar tooth development. Dev Dyn 228(1):105112. https://doi.org/10.1002/dvdy. 10352

60. Gostyńska KB et al (2016) Carriers with functional null mutations in LAMA3 have localized enamel abnormalities due to haploinsufficiency. Eur J Hum Genet 25(1):94-99. https://doi.org/10. 1038/ejhg.2016.136
61. Kim JW et al (2013) LAMB3 mutations causing autosomal-dominant amelogenesis imperfecta. J Dent Res 92(10):899-904. https:// doi.org/10.1177/0022034513502054

62. Shinomura T, Nakamura S, Ito K, Shirasawa SI, Höök M, Kimura JH (2008) Adsorption of follicular dendritic cell-secreted protein (FDC-SP) onto mineral deposits: Application of a new stable gene expression system. J Biol Chem 283(48):33658-33664. https:// doi.org/10.1074/jbc.M800719200

63. Benedete APS, Sobral APV, Lima DMC, Kamibeppu L, Soares FA, Lourenço SV (2008) Expression of transforming growth factor- $\beta 1, \beta 2$, and $\beta 3$ in human developing teeth: immunolocalization according to the odontogenesis phases. Pediatr Dev Pathol 11(3):206-212. https://doi.org/10.2350/07-09-0333.1

64. Subramanian A et al (2005) Gene set enrichment analysis: a knowledge-based approach for interpreting genome-wide expression profiles. Proc Natl Acad Sci U S A 102(43):15545-15550. https://doi.org/10.1073/pnas.0506580102

65. Tanase S, Bawden JW (1996) The immunohistochemical localization of stat-2, -3, -4 and -5 during early enamel and dentine formation in rat molars. Arch Oral Biol 41(12):1149-1160. https://doi. org/10.1016/S0003-9969(96)00084-2

66. Zhang Y et al (2019) SATB1 establishes ameloblast cell polarity and regulates directional amelogenin secretion for enamel formation. BMC Biol 17(1):104. https://doi.org/10.1186/ s12915-019-0722-9

67. Liu X et al (2019) RUNX2 contributes to TGF- $\beta 1$-induced expression of Wdr72 in ameloblasts during enamel mineralization. Biomed Pharmacother 118:109235. https://doi.org/10.1016/j. biopha.2019.109235

68. Jensen BL, Kreiborg S (1990) Development of the dentition in cleidocranial dysplasia. J Oral Pathol Med 19(2):89-93. https:// doi.org/10.1111/J.1600-0714.1990.TB00803.X

69. Xu P et al (2018) Fox proteins are modular competency factors for facial cartilage and tooth specification. Development 145(12):dev165498. https://doi.org/10.1242/dev.165498

70. Vastardis H, Karimbux N, Guthua SW, Seidman JG, Seidman CE (1996) A human MSX1 homeodomain missense mutation causes selective tooth agenesis. Nat Genet 13(4):417-421. https://doi.org/ $10.1038 / n g 0896-417$

71. Kawasaki $\mathrm{K}$ et al (2015) Expression of sox genes in tooth development. Int J Dev Biol 59(10-12):471-478. https://doi.org/10.1387/ ijdb.150192ao

72. Kodama Y et al (2019) Hmga2 regulation of tooth formation and association with Sox 2 and Nanog expression. Biochem Biophys Res Commun 509(4):1008-1014. https://doi.org/10.1016/j.bbrc. 2019.01 .017

73. Cao J et al (2019) The single-cell transcriptional landscape of mammalian organogenesis. Nature 566(7745):496-502. https:// doi.org/10.1038/s41586-019-0969-x

74. Mitsiadis TA, Drouin J (2008) Deletion of the Pitx 1 genomic locus affects mandibular tooth morphogenesis and expression of the Barx1 and Tbx1 genes. Dev Biol 313(2):887-896. https://doi.org/ 10.1016/j.ydbio.2007.10.055

75. Lacruz RS et al (2012) Identification of novel candidate genes involved in mineralization of dental enamel by genome-wide transcript profiling. J Cell Physiol 227(5):2264-2275. https://doi.org/ $10.1002 /$ jcp. 22965

76. Smith CEL et al (2017) Amelogenesis imperfecta; genes, proteins, and pathways. Front Physiol 8:435. https://doi.org/10.3389/fphys. 2017.00435

77. Jolma A et al (2015) DNA-dependent formation of transcription factor pairs alters their binding specificity. Nature 527(7578):384 388. https://doi.org/10.1038/nature15518

78. Szklarczyk D et al (2019) STRING v11: protein-protein association networks with increased coverage, supporting functional 
discovery in genome-wide experimental datasets. Nucleic Acids Res 47(D1):D607-D613. https://doi.org/10.1093/nar/gky1131

79. Li X, Zhang S, Zhang Z, Guo W, Chen G, Tian W (2019) Development of immortalized Hertwig's epithelial root sheath cell lines for cementum and dentin regeneration 06 biological sciences 0601 biochemistry and cell biology. Stem Cell Res Ther 10(1):3. https:// doi.org/10.1186/s13287-018-1106-8

80. Denes BJ, Ait-Lounis A, Wehrle-Haller B, Kiliaridis S (2020) Core matrisome protein signature during periodontal ligament maturation from pre-occlusal eruption to occlusal function. Front Physiol 11:174. https://doi.org/10.3389/fphys.2020.00174

81. About I, Laurent-Maquin D, Lendahl U, Mitsiadis TA (2000) Nestin expression in embryonic and adult human teeth under normal and pathological conditions. Am J Pathol 157(1):287-295. https:// doi.org/10.1016/S0002-9440(10)64539-7

82. Hilkens P et al (2013) Effect of isolation methodology on stem cell properties and multilineage differentiation potential of human dental pulp stem cells. Cell Tissue Res 353(1):65-78. https://doi. org/10.1007/S00441-013-1630-X

83. Merckx G et al (2020) Angiogenic effects of human dental pulp and bone marrow-derived mesenchymal stromal cells and their extracellular vesicles. Cells 9(2):312. https://doi.org/10.3390/ CELLS9020312

84. Kobayashi-Kinoshita S, Yamakoshi Y, Onuma K, Yamamoto R, Asada Y (2016) TGF- $\beta 1$ autocrine signalling and enamel matrix components. Sci Rep 6:33644. https://doi.org/10.1038/srep33644

85. Niwa $T$ et al (2018) The dynamics of TGF- $\beta$ in dental pulp, odontoblasts and dentin. Sci Rep 8(1):4450. https://doi.org/10. 1038/S41598-018-22823-7

86. Sampaziotis F et al (2021) Cholangiocyte organoids can repair bile ducts after transplantation in the human liver. Science 371(6531):839-846. https://doi.org/10.1126/science.aaz6964
87. Gjorevski $\mathrm{N}$ et al (2016) Designer matrices for intestinal stem cell and organoid culture. Nature 539(7630):560-564. https://doi.org/ 10.1038/NATURE20168

88. Zhang Y et al (2020) Polyisocyanide hydrogels as a tunable platform for mammary gland organoid formation. Adv Sci 7(18):2001797. https://doi.org/10.1002/ADVS.202001797

89. Vennekens A et al (2021) Interleukin-6 is an activator of pituitary stem cells upon local damage, a competence quenched in the aging gland. PNAS 118(25):e2100052118

90. Geraerts M, Micheils M, Baekelandt V, Debyser Z, Gijsbers R (2005) Upscaling of lentiviral vector production by tangential flow filtration. J Gene Med 7(10):1299-1310. https://doi.org/10.1002/ jgm.778

91. Bronckaers A, Hilkens P, Wolfs E, Lambrichts I (2021) By the skin of your teeth: a subcutaneous mouse model to study pulp regeneration. Methods Mol Biol 2206:223-232

92. Butler A, Hoffman P, Smibert P, Papalexi E, Satija R (2018) Integrating single-cell transcriptomic data across different conditions, technologies, and species. Nat Biotechnol 36(5):411-420. https:// doi.org/10.1038/nbt.4096

93. Liberzon A, Birger C, Thorvaldsdóttir H, Ghandi M, Mesirov JP, Tamayo P (2015) The molecular signatures database hallmark gene set collection. Cell Syst 1(6):417-425. https://doi.org/10. 1016/j.cels.2015.12.004

Publisher's Note Springer Nature remains neutral with regard to jurisdictional claims in published maps and institutional affiliations. 